Portland State University

PDXScholar

\title{
A Numerical Investigation of Microgravity Evaporation
}

Daniel Peter Ringle

Portland State University

Follow this and additional works at: https://pdxscholar.library.pdx.edu/open_access_etds

Part of the Aerodynamics and Fluid Mechanics Commons, and the Mechanical Engineering Commons Let us know how access to this document benefits you.

\section{Recommended Citation}

Ringle, Daniel Peter, "A Numerical Investigation of Microgravity Evaporation" (2020). Dissertations and Theses. Paper 5439.

https://doi.org/10.15760/etd.7312

This Thesis is brought to you for free and open access. It has been accepted for inclusion in Dissertations and Theses by an authorized administrator of PDXScholar. Please contact us if we can make this document more accessible: pdxscholar@pdx.edu. 
A numerical investigation of microgravity evaporation

by

Daniel Peter Ringle

A thesis submitted in partial fulfillment of the requirements for the degree of

Master of Science

in

Mechanical Engineering

Thesis Committee:

Dr. Mark Weislogel

Dr. Gerald Recktenwald

Dr. Mark Macdonald

Portland State University

2020 


\section{Table of Contents}

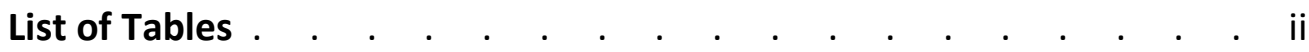

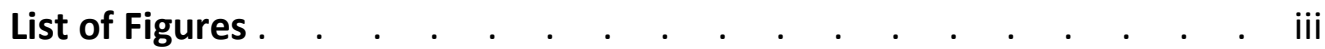

Abstract . . . . . . . . . . . . . . . . . . . . . . . $v$

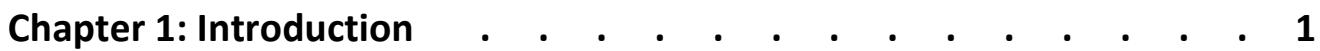

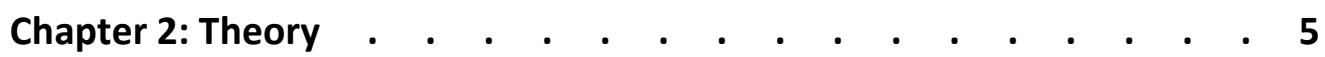

2.1 Multiphase Flows . . . . . . . . . . . . 5

2.2 Volume of Fluid . . . . . . . . . . . . . . . . 6

2.3 Fluid Film . . . . . . . . . . . . . . . . 9

2.4 Surface Tension . . . . . . . . . . . . . . . 11

Chapter 3: Evaporation . . . . . . . . . . . . . . 15

3.1 Evaporation . . . . . . . . . . . . . . . . 16

3.2 Volume of Fluid Evaporation . . . . . . . . . 19

3.3 Fluid Film Evaporation . . . . . . . . . . . 21

3.4 Stefan Tube . . . . . . . . . . . . . . . . 24

Chapter 4: Modelling . . . . . . . . . . . . . . . . . . . 26

4.1 Pre-Processing . . . . . . . . . . . . . 26

4.2 Simulating Physics . . . . . . . . . . . . . 30

4.3 Post-Processing . . . . . . . . . . . . 37

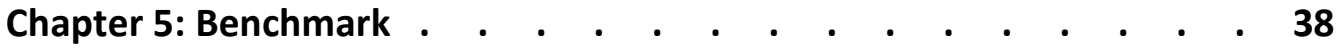

Chapter 6: Results . . . . . . . . . . . . . . . . . . 41

6.1 Numerical and Experimental Comparisons . . . . 42

6.2 Surface Area vs. Contact Line Length . . . . . . . 44

6.3 Terrestrial and Reduced Gravity Environments . . . . 49

6.4 Weighted Stefan Tube . . . . . . . . . . 51

6.5 Runtime . . . . . . . . . . . . . . . 60

Chapter 7: Conclusion . . . . . . . . . . . . . . . 61 


\section{Tables}

4.1 Thermophysical properties . . . . . . . . . . . . . . . . . . 35

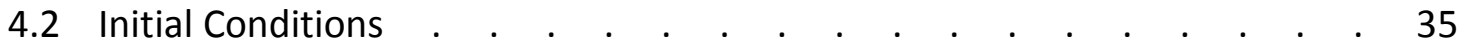

4.3 Boundary Conditions . . . . . . . . . . . . . . . . . . . 36

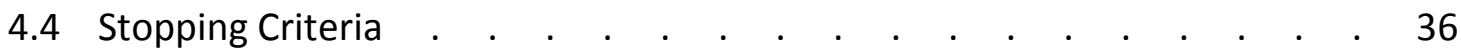

5.1 Fill level vs. Evaporation rate for the Analytical solution and the Volume of Fluid model . . . . . . . . . . . . . . . . . . . . . . . . . . . 40

6.1 Star CCM+ Evaporation rates, Square and Triangle, 95\% fill and 75\% fill . . . 42

6.2 CSELS Foam: 1-g0 vs. Microgravity evaporation rates . . . . . . . 50

6.3 CapEvap CSELS Foam block evaporation temperatures: Min, Max, Mean, V = $1 \mathrm{~cm} / \mathrm{s}$, and $5 \mathrm{~cm} / \mathrm{s}$ in $1-g 0$, and microgravity . . . . . . . . . 50

6.4 Stefan Tube weighted by Area and Contact line . . . . . . . . . 53

6.5 Slope of experimental data and Equation \#\# in linear region (Figure 6.8) . 53

6.6 CSELS Pore evaporation times and CFD heat transfer model results . . . 58

6.7 Run time and number of cores used in simulations . . . . . . . . . 60 


\section{Figures}

1.1 (a): Foam block $-40 \mathrm{~mm} \times 40 \mathrm{~mm} \times 10 \mathrm{~mm}$, (b): Pores . . . . . . 3

2.1 Multiphase flow: (a)-dispersed, (b)-Stratified . . . . . . . . 6

2.2 Equilateral Triangular Sectional Container filled 50\%, with wetting angles (a) $\Theta=60^{\circ}$, (b) $90^{\circ}$, and (c) $120^{\circ}$ computed using SE-FIT [5] . . . . . . 11

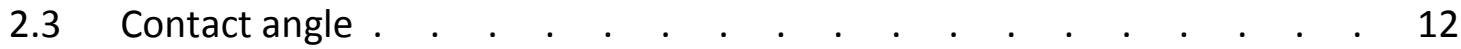

2.4 Equilateral Triangular Sectional Container filled 50\%, with wetting angles (a) $\Theta=50^{\circ}$, and (b) $70^{\circ}$ computed using SE-FIT $\quad . \quad$. . . . . . . . . 13

3.1 Stefan Tube . . . . . . . . . . . . . . . . . . . . . 23

3.2 Radius of meniscus vs. depth of capillary tube . . . . . . . . . . . 24

4.1 CSELS Triangular Pore - $10 \mathrm{~mm}$ deep with a cross-sectional area of $25 \mathrm{~mm}^{2} \quad 27$

4.2 Regions layout $\quad . \quad$.

4.3 Polyhedral mesh: Triangle Geometry, $95 \%$ fill $\quad$. . . . . . . . . . $\quad 29$

4.4 Interface mesh density and Prism layers generated at solid boundaries . 30

5.1 Stefan Tube: $Z=50 \mathrm{~mm}$, Fill levels $=25 \mathrm{~mm}, 31.25 \mathrm{~mm}, 37.5 \mathrm{~mm}, 43.75$ $\mathrm{mm}, \mathrm{A}_{\mathrm{c}}=7.854 \mathrm{E}-7 \mathrm{~m}^{2}, \mathrm{~V}_{\mathrm{in}}=1 \mathrm{~m} / \mathrm{s}, \mathrm{P}_{\text {out }}=1$ atmosphere . . . . . . 39

5.2 Fill level vs. Evaporation rate for equation 5.1 and the Volume of Fluid evaporation model . . . . . . . . . . . . . . . . . . . . 39

6.1 Experimental results: Mass of water $(\mathrm{kg})$ as a function time (s) for (a) triangular pore geometry and (b) square pore geometry . . . . . . 43

6.2 (a): Evaporation rate along section A-A in triangular geometry with a $75 \%$ fill fraction, (b): Cross-sectional view showing volume of fraction of water from triangular pore with a $75 \%$ fill , (c): Cross-sectional view showing mass 
fraction of water above free surface from triangular pore with a 75 percent

fill

6.3 (a): Evaporation rate along Section A-A, (b): Boundary layer over CapEvap triangular geometry, $100 \%$ fill level . . . . . . . . . . . . 48

6.4 Numerical Evaporation rate of free surface: (a) CapEvap Square Geometry, 75\% fill level. (b) CapEvap Triangle Geometry, 75\% fill level. Parallax due to perspective . . . . . . . . . . . . . . . . . . . . . 48

6.5 Surface temperature of CapEvap CSELS Foam during evaporation, $V=5$

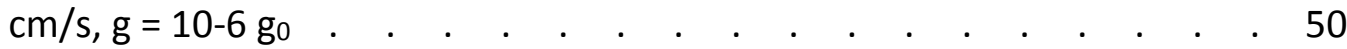

6.6 Semi-Log plot of Ri vs. airflow velocity for CapEvap Foam Geometry 51

6.7 Position of free surface as a function of time compared with equations 3.29, 3.31 and 3.32 for (a): Square pore geometry, and (b): Triangular pore geometry . . . . . . . . . . . . . . . . . . . . 52

6.8 Linear region evaporation: Experimental data vs Equation 3.32 for (a) square pore geometry, and (b) triangular pore geometry $\quad . \quad$. . . $\quad$. 54

6.9 Single-piece 3-D printed CSELS CapEvap device . . . . . . . . . . 56

6.10 (a): Heat flux to free surface, (b): Temperature profile mid-line of pores, (c): Temperature profile Pores and support . . . . . . . . . . 56

6.11 Time lapse pores from Figure 1.1 (b) undergoing evaporation . . . . 59 


\section{Abstract}

Evaporation is important to myriad engineering processes such as cooling, distillation, thin film deposition, and others. In fact, NASA has renewed interest in using cabin air pressure evaporation as a means to recycle waste water in space. As one example, NASA recently conducted experiments aboard the International Space Station (ISS) to measure evaporation rates in microgravity and to determine the impacts of porous structure on the process. It has long been assumed that differences in evaporation rates between 1-go and microgravity are small. However, discrepancies by as much as $40 \%$ have been observed in practice. The assumption now is that such differences are not only due to a lack of buoyancy in the vapor phase in microgravity $\left(10^{-6} \mathrm{go}\right)$, but also to pore geometries, wetting conditions, and airflow. Numerical models are developed herein to assess the viability of Star CCM+ as a CFD tool to accurately model such phenomena, as well as to identify what mechanisms are responsible for the difference observed in practice between 1-go and microgravity. The code is benchmarked via comparisons to Stefan Tube analytical solutions with agreement to within approximately $\pm 1 \%$. Accounting for pore 
geometry, comparisons to NASA ISS flight data yields results accurate to $\pm 14 \%$. Additionally, the analytical solution to the Stefan Tube is weighted for both the actual free surface area and contact line length yielding results accurate to $4.4 \%$ and $6.1 \%$ depending on pore geometry.

The CFD models are able to identify the mechanisms responsible for the effects of microgravity on the rate of evaporation and it is shown that these effects can be minimized and even wholly negated by sufficiently high airflow velocity. 


\section{Chapter 1}

\section{Introduction}

Optimizing resource utilization aboard spacecraft is critical for long duration space exploration. Increased waste-water reclamation (i.e., urine, humidity condensate, etc.) provides a case in point. Currently, waste-water is reclaimed at a rate of $80 \%$ [1]. However, for human missions to Mars, NASA's goal is to reclaim greater than $98 \%$. Similar to atmospheric pressure desalination processes on Earth, spacecraft cabin pressure evaporation is under consideration as a passive means to recycle waste-water. Surprisingly, evaporation in microgravity is not a well-researched field. Studies conducted to date have focused on short duration, high temperature evaporation of suspended drops in drop tower experiments [2]. Unfortunately, such investigations are unrepresentative of the slow evaporative processes anticipated for ambient waste-water distillation within porous structures, conduits, and media. 
In 2016, NASA's Johnson Space Center initiated a fast-to-flight engineering demonstration for capillary-based technologies aboard the International Space Station (ISS). The Capillary Structures for Exploration Life Support experiment (CSELS) consists of three related experiments, one of which focuses primarily on the fluid mechanics and transport of a brine water-recovery system and seeks to quantify evaporation rates of target fluids at ambient temperatures in the microgravity environment of an orbiting spacecraft [3]. These experiments are called the CSELS CapEvap experiments (for capillary evaporation). For the CSELS CapEvap experiments the evaporation rates were measured using two approaches. Figure 1.1 (a) shows a second experimental geometry where a foam block is connected to a tube serving as a water reservoir to visually measure evaporation rates from the foam in microgravity. As evaporation from the foam takes place, water is drawn into the foam from the tube maintaining the foam at $100 \%$ saturation until the tube is depleted. This allows for easily and accurately measuring the evaporation rate aboard the ISS. Figure 1.1 (b) shows a series of 3-D printed transparent pores of varying cross-sectional geometry with fixed height and cross-sectional area. The individual pores are filled by syringe by the onboard crew and placed in the Japanese Experimental Module (JEM) of the ISS where time lapse images were collected over numerous days of evaporation. All pores initially have the same free surface area, however, contact line length varies due to cross-sectional geometry. As evaporation occurs both the free surface area and the contact line length change due to the different cross-sectional geometries and wetting conditions which allows for the unique isolation 
of such effects. Additionally, $66 \%$ of the airflow aboard the ISS is between 5 and $20 \mathrm{~cm} / \mathrm{s}$. The experiments were placed in a 'quiet' location. Given this information, and with no air flow measurements available, the low end of the range given is used in the numerical models of the CSELS CapEvap pores experiment.

The results from the CapEvap experiments show that as the surface area and contact line increase, so too does the rate of evaporation, and evaporation in $1-g_{0}$ is as much as $40 \%$ greater than evaporation in microgravity for similar temperature, pressure, air flow, and relative humidity conditions. Understanding the relative contributions of surface area, contact line length, and pore geometry as well as the mechanisms most sensitive to the presence and absence of gravity are of immediate practical interest and requires further investigation.

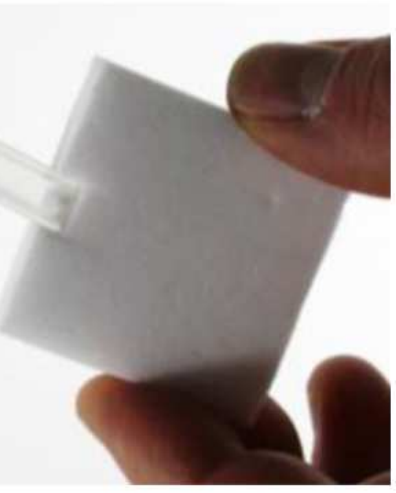

(a)

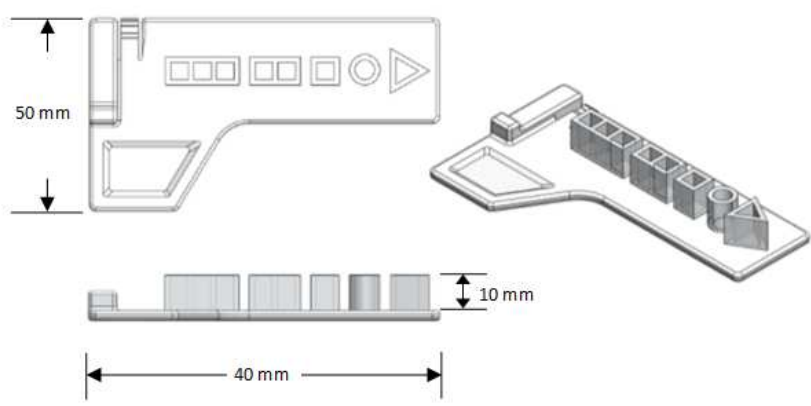

(b)

Figure 1.1 - (a): Foam block-40mm X 40mm X10mm, (b): Pores 
The resources necessary to conduct experiments aboard the ISS, notably time and money, are considerable. For this reason, there is value in numerically modelling myriad microgravity flows of interest. In this study the commercial code Star $\mathrm{CCM}+$ is employed to simulate the CSELS CapEvap experiments via CFD. The success of the numerical model is assessed by benchmarks with analytical solutions where available and by validation with the terrestrial and spaceflight experiments. 


\section{Chapter 2}

\subsection{Multiphase Flows}

Multiphase fluid flows are flows characterized by more than one fluid phase. Water and humid air constitute a multiphase flow. Multiphase flows are often classified by the distribution of phases present within the flow as the phase distribution can vary considerably. However, multiphase flows largely belong to one of two categories: separated or dispersed as depicted in Figure 2.1. Furthermore, separated multiphase flows may or may not be stratified. This distinction is important as flows that would otherwise stratify in the 1-go environment of earth may not stratify in the absence of significant buoyant forces in space. For this reason, and for a variety of critical engineering applications aboard spacecraft, special care must be taken to prevent vapor phases from becoming dispersed within liquid phases. The flows modeled in this study $\left(1-g_{0}\right.$ and microgravity) fall under the classification of separated multiphase flows. 


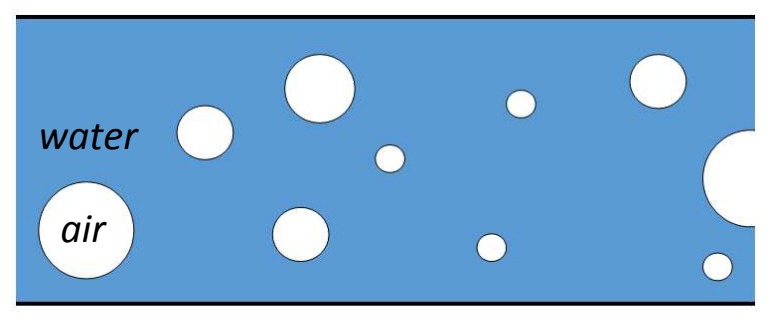

(a)



(b)

Figure 2.1 - Multiphase flow: (a) - dispersed, (b) - Stratified

\subsection{Volume of Fluid}

The Volume of Fluid (VOF) method, first published in 1981 by Hirt and Nichols [4], is an interface Tracking CFD algorithm belonging to the family of Eulerian methods. This model requires that the fluid phases be immiscible and that a clearly defined interface is present. VOF is well-suited to numerically model evaporation at the liquid free surface. For every finite volume, or cell, containing either of the two phases, a volume fraction $\alpha$ is calculated such that

$$
\alpha=\frac{V_{l}}{V}
$$

where $V_{l}$ is the volume of the liquid phase and $V$ is the total volume of the cell. When $\alpha=$ 0 , the cell contains only the gas phase and when $\alpha=1$ the cell contains only the liquid phase. When $0<\alpha<1$ the free surface resides somewhere within the cell. For cells 
containing the free surface, new fluid properties must be calculated for density $\rho$, viscosity $\mu$, and specific-heat $C_{p}$. The weighted volume fractions are

$$
\begin{gathered}
\rho=\sum_{i} \rho_{i} \alpha_{i} \\
\mu=\sum_{i} \mu_{i} \alpha_{i} \\
c_{p}=\sum_{i} \frac{c_{p_{i}} \rho_{i}}{\rho} \alpha_{i} .
\end{gathered}
$$

\section{Governing Equations}

Because VOF accounts for multiple phases simultaneously, the governing equations must be reformulated to account for the presence of multiple phases. The governing equations used by STAR-CCM+, modified specifically for VOF are introduced here. The momentum equation is

$$
\begin{gathered}
\frac{\partial}{\partial \mathrm{t}}\left(\int_{V} \rho \mathbf{v} d V\right)+\oint A \oint_{A} \rho \mathbf{v} \otimes \mathbf{v} \cdot d \mathbf{a}= \\
-\oint_{A} p \mathbf{I} \cdot d \mathbf{a}+\oint_{A} \mathbf{T} \cdot d \mathbf{a}+\int_{V} \rho \mathbf{g} d V+\int_{V} \mathbf{f} b d V \\
-\sum_{i} \int_{A} \alpha_{i} \rho_{i} \mathbf{v}_{\boldsymbol{d}, \boldsymbol{i}} \otimes \mathbf{v}_{\boldsymbol{d}, \boldsymbol{i}} \cdot d \mathbf{a},
\end{gathered}
$$

where $p$ is the pressure, $\boldsymbol{I}$ is the unity tensor, $\boldsymbol{T}$ is the stress tensor, and $\boldsymbol{f}_{b}$ is the vector of body forces. 
Mass conservation equation is given by

$$
\frac{\partial}{\partial \mathrm{t}}\left(\int_{V} \rho d V\right)=+\oint_{\boldsymbol{A}} \rho \mathbf{v} \cdot d \mathbf{a}=\int_{V} S d V
$$

where $S$ is a mass source term related to the phase source term such that

$$
S=\sum_{i} S_{a i} \cdot \rho_{i}
$$

Conservation of energy is given by

$$
\begin{aligned}
\frac{\partial}{\partial \mathrm{t}} \int_{V} \rho E d V+ & \oint_{A}\left[\rho \mathrm{H} \mathbf{v}+p+\sum_{i} \alpha_{i} \rho_{i} H_{i} \mathbf{v}_{d, i}\right] \cdot d \mathbf{a} \\
& =-\oint_{A} \dot{\mathbf{q}}^{\prime \prime} \cdot d \mathbf{a}+\oint_{\boldsymbol{A}} \mathbf{T} \cdot \mathbf{v} d \mathbf{a}+\int_{\boldsymbol{V}} \mathbf{f}_{\boldsymbol{b}} \cdot \mathbf{v} d V+\int_{V} S_{E} d V
\end{aligned}
$$

where $E$ is the total energy, $H$ is the total enthalpy, $\dot{\mathbf{q}}^{\prime \prime}$ is the heat flux vector, and $S_{E}$ is a user-defined energy source term. The phase fraction transport equation is given by

$$
\begin{aligned}
\frac{\partial}{\partial \mathrm{t}} \int_{V} \alpha_{i} d V+\oint_{A} \alpha_{i} \mathbf{v} \cdot d \mathbf{a} & \\
& =\int_{V}\left(S_{\alpha_{i}}-\frac{\alpha_{i}}{\rho_{i}} \frac{D \rho_{i}}{D t}\right) d V-\int_{V} \frac{1}{\rho_{i}} \nabla \cdot\left(\alpha_{i} \rho_{i} \mathbf{v}_{\boldsymbol{d}, \boldsymbol{i}}\right) d V
\end{aligned}
$$

where $\mathbf{a}$ is the surface area vector, $\mathbf{v}$ is the mass averaged velocity, $\mathbf{v}_{\boldsymbol{d}, \boldsymbol{i}}$ is the diffusion velocity, $S_{\alpha_{i}}$ is the user defined source term of phase $i$ and $\frac{D \rho_{i}}{D t}$ is the material derivative of $\rho_{i}$. 


\subsection{Fluid Film}

The CSELS CapEvap foam evaporation test employs a saturated foam block that exploits surface tension to continuously pump liquid to the foam surface from a tube reservoir to replace the liquid lost to evaporation. The net effect is that the foam maintains essentially a saturated state modelled as a thin fluid film on its surface that undergoes evaporation. Star $\mathrm{CCM}+$ has a fluid film model that is well-suited to simulate this behavior. The governing equations for this model are provided here with unique quantities identified.

\section{Governing Equations}

The mass conservation equation is

$$
\frac{\partial}{\partial t} \int_{V} \rho_{f} d V+\int_{A} \rho_{f} \boldsymbol{V}_{f} \cdot d \boldsymbol{a}=\int_{V} \frac{S_{u}}{h_{f}} d V
$$

where $\rho_{f}$ is the film density, $\boldsymbol{V}_{f}$ is the film velocity, $S_{u}$ is the mass source/sink per unit area, and $h_{f}$ is the film thickness. The momentum Equation is

$$
\begin{aligned}
\frac{\partial}{\partial t} \int_{V} \rho_{f} \mathbf{v}_{f} d V & +\int_{A} \rho_{f} \mathbf{v}_{f} \otimes \mathbf{v}_{f} \cdot d \mathbf{a} \\
& =\int_{A} \mathbf{T}_{f} \cdot d \mathbf{a}-\int_{A} p_{f} d \mathbf{a}+\int_{V}\left(\mathbf{f}_{b}+\frac{\mathbf{s}_{m}}{h f}\right) d V
\end{aligned}
$$

where $\mathbf{s}_{m}$ is the momentum source, $p_{f}$ is the pressure, $\mathbf{f}_{b}$ is the body force, and $\mathbf{T}_{f}$ is the viscous stress tensor in the film. The kinematic and dynamic conditions at the interface between the film and the surrounding fluid are satisfied by 


$$
\begin{gathered}
\left(\mathbf{v}_{f}\right)_{\text {int }}=(\mathbf{v})_{\text {int }}, \\
\left(\mathbf{T}_{f} \cdot d \mathbf{a}+p_{f} d \mathbf{a}\right)_{i n t}=(\mathbf{T} \cdot d \mathbf{a}+p d \mathbf{a})_{i n t},
\end{gathered}
$$

where the subscript $f$ denotes the fluid film and no subscript denotes the surrounding fluid. Assuming that the normal components of the viscous and convective terms are negligible, the pressure distribution within the fluid film is

$$
p_{f}(\xi)=p_{i n t}-\boldsymbol{s}_{m} \cdot \boldsymbol{n}-\rho_{f} \boldsymbol{f}_{b} \cdot \boldsymbol{n}\left(h_{f}-\xi\right)+\int_{\xi}^{h_{f}} \frac{d}{d t}\left(\rho_{f} \mathbf{v}_{f} \cdot \boldsymbol{n}\right) d \xi
$$

where $\boldsymbol{n}$ is the wall surface unit vector pointing towards the film and $\xi$ is the local wall coordinate. $\boldsymbol{s}_{m}$ is applied at the film surface. The energy equation is

$$
\begin{aligned}
\frac{\partial}{\partial \mathrm{t}} \int_{V} \rho_{f} E_{f} d V & +\int A\left[\rho_{f} H_{f} \mathbf{v}_{f} \cdot d \mathbf{a}\right. \\
& =\int_{A} \mathbf{q}^{\prime \prime}{ }_{f} \cdot d \mathbf{a}+\int_{A} \mathbf{T}_{f} \cdot \mathbf{v}_{f} d \mathbf{a}+\int_{V} \mathbf{f}_{b} \cdot \mathbf{v}_{f} d V \\
& +\int_{V} \frac{S_{E}}{h_{f}} d V
\end{aligned}
$$

and species mass conservation is maintained via

$$
\frac{\partial}{\partial t} \int_{V} \rho_{f} Y_{i, f} d V+\int_{A} \rho_{f} \mathbf{v}_{f} Y_{i, f} \cdot d \boldsymbol{a}=\int_{A}\left(\frac{\mu_{f}}{\sigma}\right) \nabla Y_{i, f} \cdot d \boldsymbol{a}+\int_{V} \frac{S_{u, i}}{h_{f}} d V .
$$

The volume of the fluid film is subtracted from the volume of the gas phase in adjacent cells. The volume fraction is computed as 


$$
\alpha_{f}=\min \left(\frac{V_{f}}{V}, \alpha_{f, \max }\right)
$$

where $V_{f}$ is the volume of the film, $V$ is the volume of the cell, and $\alpha_{f, \max }$ is the maximum volume fraction of the film.

\subsection{Surface Tension}

Surface tension impacts the shape, stability, and general behavior of free surfaces in myriad engineering applications. Surface tension $\sigma$ is the effective result of cohesive forces existing between molecules in the liquid phase and adhesive forces between the liquid-gas and liquid-solid phases.

The free surface shape is determined by the surface tension, container geometry, and the adhesive forces existing between the liquid-solid and gas-solid as shown in Figure 2.2.

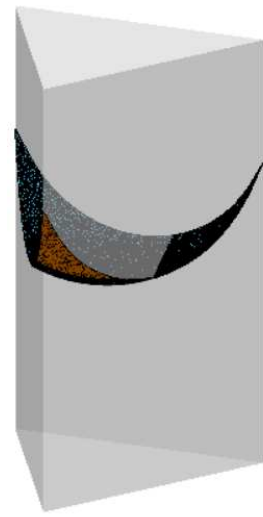

(a)

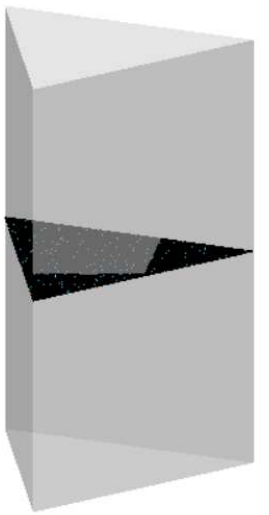

(b)

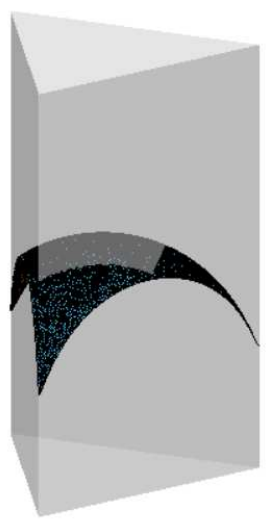

(c)

Figure 2.2 - Equilateral Triangular Sectional Container filled 50\%, with wetting angles (a) $\theta=60^{\circ}$, (b) $90^{\circ}$, and (c) $120^{\circ}$ computed using SE-FIT [5] 
The degree to which a liquid adheres to a surface can be characterized as the wettability of the surface and is determined by the balance between the adhesive and cohesive forces. Such wettability can be quantified by the contact angle $\theta$ between the liquid-gas interface and the liquid-solid interface (Figure 2.3). For $0^{\circ}<\theta<90^{\circ}$ the surface is considered wetting. For $90^{\circ}<\theta<180^{\circ}$ the surface is considered non-wetting.



Figure 2.3 - Contact angle

Additionally, when the Concus-Finn condition is satisfied, namely $\theta<\frac{\pi}{2}-\alpha$, where $\alpha$ is the half angle of the interior corner, the fluid will remain pinned at the opening of the pores in the corner regions (Figure 2.4) such that a rivulet will remain along the interior corner as the bulk liquid recedes during evaporation. 


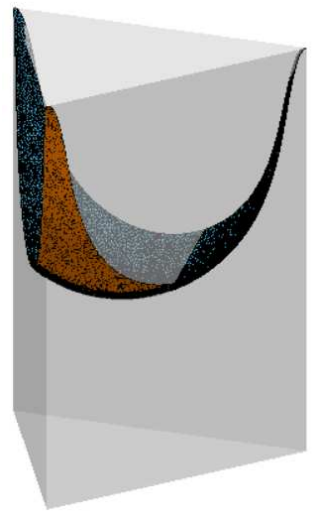

(a)

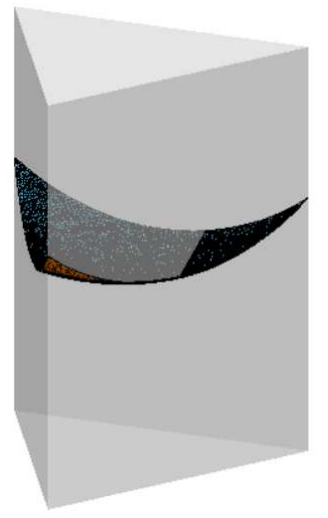

(b)

Figure 2.4 - Equilateral Triangular Sectional Container filled 50\%, with wetting angles (a) $\theta=50^{\circ}$, and (b) $70^{\circ}$ computed using SE-FIT

In the absence of gravity, the liquid free surface shape is determined by the surface tension, contact angle, and system geometry. The relative impact of surface tension and gravity on the surface geometry can be determined by a dimensionless ratio defined as the Bond number, $B o \equiv \rho g R^{2} / \sigma$, where $\rho$ is the fluid density and $R$ is the characteristic surface length scale. When $B o \ll 1$, surface configurations are those of constant curvature that again depend strongly boundary conditions. The following formulations are employed to solve for the static free surface configuration using the SEFIT software [5] and dynamic free surface flows using Star CCM+. The SE-FIT software employs K. Brakke's Surface Evolver algorithm [6] as a kernel to resolve for minimum surface energy state of the system by employing the gradient descent method. The numerical formulation of surface tension used in Star $\mathrm{CCM}+$ is based upon the Continuum 
Surface Force (CSF) method first developed by Brackbill et al. CSF calculates the normal vector $\boldsymbol{n}$ as the gradient of the smooth field of phase volume fraction $\alpha_{i}$,

$$
\boldsymbol{n}=\boldsymbol{\nabla} \alpha_{i}
$$

and the curvature is the divergence of the unit normal vector $\boldsymbol{n}$,

$$
\kappa=-\boldsymbol{\nabla} \cdot \frac{\alpha_{i}}{\left|\alpha_{i}\right|} .
$$

For the models in this paper the surface tension of water is defined as a constant and is given by $\sigma=0.072 \mathrm{~N} / \mathrm{m}$. 


\section{Chapter 3}

\section{Evaporation}

Evaporation is the process of a liquid transitioning into the gas phase at the liquid/vapor interface while condensation is the reverse of this process. As evaporation and condensation will typically occur simultaneously, the rate of evaporation must be greater than that of condensation for net evaporation to occur. A useful measure for determining whether or not evaporation or condensation will occur is the relative humidity, $\phi=P_{h_{2} o} / P^{*}{ }_{h_{2} o}$, where $P_{h_{2} o}$ is the partial pressure of water vapor in the air and $P^{*}{ }_{h_{2} O}$ is the equilibrium vapor pressure of water vapor in the air. When this ratio is less than one, net evaporation occurs. During the process of evaporation only molecules with sufficient kinetic energy can escape the liquid phase which results in the average kinetic energy of the molecules at the free surface being reduced as a result of evaporation and a temperature drop is observed in the liquid. This thermal fluid property is called evaporative cooling. As water molecules must have sufficient kinetic energy to 
undergo evaporation, the amount of energy necessary to vaporize a specific mass of liquid is the latent heat of vaporization, $\Delta h_{v a p}$. The rate at which this process occurs is a function of both the temperature and vapor pressure whereby increasing the temperature or lowering the relative vapor pressure (humidity) will both result in increasing the rate of evaporation. As evaporation occurs, the humidity above the free surface increases resulting in slower evaporation rates. Air flow over the free surface will help to reduce the humidity by continuously replacing the cooler humid air above the free surface with air at ambient temperature and humidity resulting in increased evaporation. In a gravitydominated environment (i.e., Earth), buoyancy helps to drive fluid motion through natural convection. However, in the microgravity environment of orbiting spacecraft, buoyancy is significantly reduced. The modelling of evaporation from pores in microgravity is accomplished herein using the Star CCM+ VOF method. Modelling the effects of gravity uses the Fluid Film Method. The analytical solution to the Stefan Tube problem is employed to benchmark the VOF evaporation model in Star CCM+.

\section{1) Volume of Fluid Evaporation}

Star CCM+ models evaporation as diffusion-driven, where the mass fraction at the interface is determined by Raoult's law which states that the vapor pressure of a mixture is equal to the product of the vapor pressure of the pure solvent at the given temperature and its mole fraction and is given by 


$$
p_{i}=\alpha_{i} p_{i}^{*}
$$

The evaporation rate is given by

$$
\dot{m}_{\imath}=-\frac{\left.\rho_{g} D_{g, i} \frac{\partial Y_{g, i}}{\partial n}\right|_{s}}{1-\sum_{j=1}^{N_{v}} Y_{g j}^{S}}
$$

where $m_{i}$ is the evaporation rate in $\frac{k g}{m^{2} s}, \rho_{g}$ is the density of the gas phase, $D_{g, i}$ is the diffusion coefficient, $Y_{S}$ is the component mass fraction at the liquid surface, and $N_{v}$ is the number of components undergoing evaporation. The mass fraction of the individual components at the surface is then the ratio of the partial pressure to the total pressure

$$
X_{i, g}^{s}=\frac{p_{i}}{p}
$$

and the conversion to mass fraction is

$$
Y_{g, i}^{S}=\frac{X_{i}^{S} W_{i}}{\sum_{j=1}^{N v} X_{j}^{S} W_{j}+\sum_{j=1}^{N_{g, p}} X_{j}^{s} W_{j}}
$$

where $N_{g, p}$ is the number of inert non-condensable components in the gas phase. The molar fraction of the inert components is unknown, but can be approximated as

$$
\sum_{j=1}^{N_{g, p}} X_{j}^{s} W_{j}=X_{b g}^{s} W_{b g}
$$


where

$$
X_{b g}^{S}=1-\sum_{j=1}^{N_{v}} X_{j}^{S}
$$

and

$$
W_{b g}=\frac{\sum_{j=1}^{N_{g, p}} X_{j} W_{j}}{\sum_{j=1}^{N_{g, p}} X_{j}}
$$

Therefore, the interfacial mass fraction may be approximated as

$$
\begin{gathered}
Y_{g, i}^{S} \approx \frac{X_{i}^{S} W_{i}}{\sum_{j=1}^{N_{v}} X_{j}^{S} W_{j}+X_{b g}^{S} W_{b g}}, \\
Y_{g, m}=\alpha_{l} Y_{g, i}^{S}+\alpha_{g} Y_{g, i}, \\
\dot{M}^{\prime}{ }_{i, c} \approx-\frac{\rho_{g} D_{g, i} \nabla Y_{g, m} \nabla \alpha_{l} V_{c}}{1-\sum_{j=1}^{N_{v}} Y_{g j}^{S}} .
\end{gathered}
$$

The equilibrium vapor pressure is a function of temperature and increases with temperature according to the Antoine equation [7].

$$
\log _{10}\left(P_{h_{2} o}^{*}\right)=A-\frac{B}{T+C}
$$

where $A, B$, and $C$ are known constants, and $T$ is the temperature. A sufficiently fine mesh is necessary to accurately capture evaporation rates. The relative error in the simulation has been shown to be proportional to 


$$
\varepsilon_{r e l} \approx \frac{1}{H|\nabla \alpha|^{\prime}}
$$

where $H$ is the thickness of the boundary layer and $|\nabla \alpha|^{-1}$ is a measure of the mesh size (Star $\mathrm{CCM}+)$.

\section{2) Fluid Film Evaporation}

The species mass flux for every $i_{\text {th }}$ component is conserved at the interface of the gas and fluid film such that

$$
\rho Y_{i}(v-\dot{h})-\rho D_{i} \frac{d Y_{i}}{d y}=\rho_{f} Y_{f, i}\left(v_{f}-\dot{h_{f}}\right)-\rho_{f} D_{f, i} \frac{d Y_{i}}{d y} \| f
$$

where, evaluated at the interface, $\rho$ and $\rho_{f}$ are the gas a liquid film densities, $Y_{i}$ and $Y_{f, i}$ are the mass fractions for the gas and liquid film, $v$ and $v_{f}$ are the normal velocity components for the gas and liquid film, $D_{i}$ and $D_{f, i}$ are the gas and liquid film molecular diffusion coefficients, and $\dot{h}$ is the rate of change of the film thickness.

A mass flux balance yields

$$
\rho(v-\dot{h})=\rho_{f}\left(v_{f}-\dot{h}\right)
$$

Combining equations 3.13 and 3.14 yields

$$
\rho_{f}\left(v_{f}-\dot{h}\right)\left(Y_{i}-Y_{f, i}\right)+\rho_{f} D_{f, i} \frac{d Y_{i}}{d y} \| f-\rho D_{i} \frac{d Y_{i}}{d y}=0
$$

where the evaporation rate is

$$
\dot{m}_{v}=-\rho_{f} \dot{h}
$$


setting $v_{f}=0$ from this point forward. Summing over all liquid film components $N_{L}$ yields

$$
\left[1-\sum_{j}^{N_{L}} Y_{j}\right] \dot{m}_{v}=-\sum_{j}^{N_{L}} \rho D_{i} \frac{d Y_{i}}{d y}
$$

where

$$
-Y_{j} \dot{m}_{v}=-\rho D_{i} \frac{d Y_{i}}{d y}
$$

for all $N_{L}$ species $i$ in the film that are inert, the summation limited to the $N_{v}$ interacting components. The total evaporation rate is then given by

$$
\dot{m}_{v}=-\frac{\sum_{j}^{N_{v}} \rho D_{i} \frac{d Y_{i}}{d y}}{1-\sum_{j}^{N_{v}} Y_{j}} .
$$

Equation \#\# is only valid below saturation where

$$
\sum_{j}^{N_{v}} Y_{j}<1
$$

The normal derivative is treated through the species transfer coefficients $s_{t, i}$ and the Spalding transfer number $B$ such that

$$
\dot{m}_{v}=-\frac{\sum_{j}^{N_{v}} s_{t, j}\left(Y_{c, j}-Y_{j}\right)}{1-\sum_{j}^{N_{v}} Y_{j}} \cdot \frac{\ln (1+B)}{B},
$$

where the subscript $c$ indicates a cell value and $B$ is defined by

$$
B \equiv \frac{\sum_{j}^{N_{v}} Y_{j}-\sum_{j}^{N_{v}} Y_{\infty}}{1-\sum_{j}^{N_{v}} Y_{j}}
$$


where it is assumed that $Y_{\infty} \approx Y_{c}$. The interfacial gas mass fraction is determined using Raoult's law as described in chapter \#\#. The interfacial heat flux balance is expressed as

$$
k_{g} \frac{d T}{d y}-k_{l} \frac{d T}{d y} \| f-\dot{Q}_{v}=0
$$

where $k$ denotes the thermal conductivity and

$$
\dot{Q_{v}}=\sum_{i}^{N_{v}} \Delta H_{i}^{\mathrm{vap}} \dot{m}_{v, i}
$$

Combining equations \#\# and \#\# yields an expression for the total evaporation rate valid at all conditions

\subsection{Stefan Tube Evaporation}

$$
\dot{m}_{v}=\frac{\dot{Q}_{v}+\sum_{i}^{N_{v}} \Delta H_{i}^{\mathrm{vap}} \rho D_{i} \frac{d Y_{i}}{d y}}{\sum_{i}^{N_{v}} \Delta H_{i}^{\mathrm{vap}} Y_{i}} .
$$

The Stefan tube is a simple device developed to measure diffusion coefficients by measuring the rate at which a liquid index recedes into a tube due to evaporation [8]. The diffusion coefficients are determined using

$$
D_{A B}=\frac{\rho_{A, L} P_{B, l m} R T}{t P M_{A}\left(P_{A, Z t}-P_{A, Z 1}\right)}\left(\frac{Z_{t}^{2}-Z_{1}^{2}}{2}\right),
$$

where $D_{A B}$ is the diffusion coefficient of Liquid A diffusing into gas $\mathrm{B}, \rho_{A, L}$ is the density of liquid $A$ at ambient temperature $T, R$ is the gas law constant, $M_{A}$ is the molar mass of liquid $A, P$ is the absolute pressure, $P_{A, Z_{1}}$ is the vapor pressure at position $Z_{1}, P_{A, Z_{t}}$ is the equilibrium vapor pressure at temperature $T, P_{B, l m}$ is the log-mean pressure difference between points $A$ and $B$ given by 


$$
P_{B, l m}=\frac{\left(P-P_{A, Z_{t}}\right)-\left(P-P_{A, Z 1}\right)}{\ln \left(\frac{P-P_{A, Z}}{P-P_{A, Z 1}}\right)},
$$

where $t$ is the elapsed time in seconds, $Z_{1}$ is the initial height of the free surface relative to the opening of the tube, and $Z_{t}$ is the height of the free surface as a function of time. With the knowledge of the diffusion coefficient $D_{A B}$, equation 3.26 can instead be solved for the height $Z_{t}$ as a function of time such that

$$
Z_{t}=\left(\frac{2 D_{A B} t P M_{A}\left(P_{A 1}-P_{A 2}\right)}{\rho_{A, L} P_{B, l m} R T}+Z_{1}^{2}\right)^{1 / 2}
$$

which by setting $Z_{1}=0$ simplifies to

$$
Z_{t}=\left(\frac{2 D_{A B} t P M_{A}\left(P_{A 1}-P_{A 2}\right)}{\rho_{A, L} P_{B, l m} R T}\right)^{1 / 2} .
$$

Further, for fluid interfaces of uniform height, the evaporation rate of the Stephan tube is the derivative of equation 3.29 with respect to time multiplied by the density of the evaporating fluid

$$
\frac{\partial Z_{t}}{\partial x}=\left(\frac{D_{A B} P M_{A}\left(P_{A 1}-P_{A 2}\right)}{2 t \rho_{A, L} P_{B, l m} R T}\right)^{1 / 2} \rho_{A, L}
$$




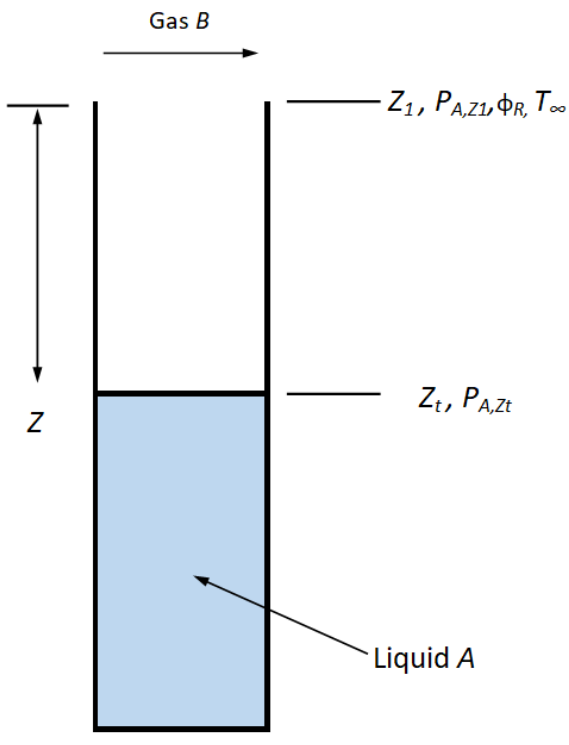

Figure 3.1 - Stefan Tube

The analytical equations above are one-dimensional and as such it is assumed that the liquid vapor interface is flat. The actual scenario is more similar to the representation given in Figure 3.2 - a meniscus of height $H$ forms due to the surface tension, wetting conditions, and geometry of the tube. Such menisci may remain at a constant shape, but they are not flat. If the height of the meniscus $H$ is much less than the depth of the liquid into the tube, $H / Z \ll 1$, the assumption of a flat interface can be made because the effectively constant curvature region of the meniscus shrinks to zero as $Z \rightarrow \infty$. Also, equations $3.26-3.30$ assume that the temperature field is uniform, which holds with increased error if temperature differences are small. 


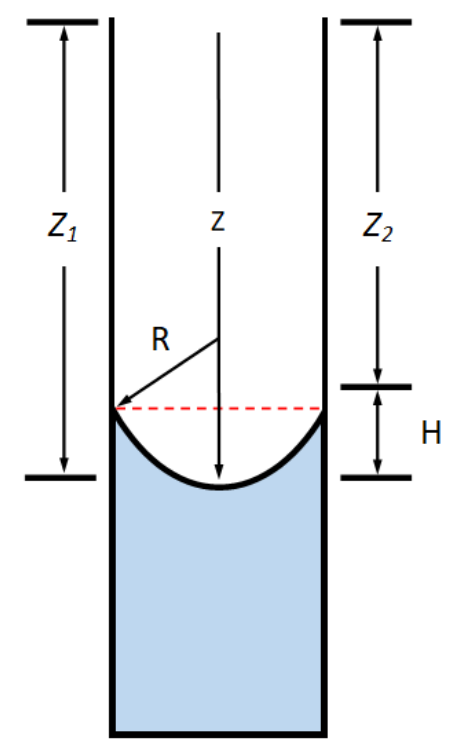

Figure 3.2 - Radius of meniscus vs. depth of capillary tube

\subsection{Weighted Stefan Tube}

The assumption $H / Z \ll 1$ may be valid for geometries and wetting conditions where the Concus-Finn condition is not met. However, when the Concus-Finn condition is satisfied, as with the CSELS CapEvap triangular pore geometry, this assumption is no longer valid. To address this situation, equation 3.26 is weighted to account for the increased surface area and contact line length. This is accomplished by multiplying the diffusion coefficient in equation 3.26 by a function that describes the surface area or the contact line length as a function of the position $Z$. These functions are determined with the use of SE-FIT:

$$
A(Z) \cdot D_{A B}=\frac{\rho_{A, L} P_{B, l m} R T}{t P M_{A}\left(P_{A, Z t}-P_{A, Z 1}\right)}\left(\frac{Z_{t}^{2}-Z_{1}^{2}}{2}\right)
$$


and

$$
C(Z) \cdot D_{A B}=\frac{\rho_{A, L} P_{B, l m} R T}{t P M_{A}\left(P_{A, Z t}-P_{A, Z 1}\right)}\left(\frac{Z_{t}^{2}-Z_{1}^{2}}{2}\right)
$$

where $A(Z)=$ Actual area/Projected area and $C(Z)=$ Actual contact line length/Projected contact line length. These equations will be validated against the experimental data to assess their viability for use in predicting evaporation rates from pores of varying geometries. 


\section{Chapter 4}

\section{Modelling}

The modelling process in Star CCM+ is comprised of three primary phases: PreProcessing, Simulating Physics, and Post-Processing. Pre-Processing involves creating the geometry, defining the region layouts, and discretizing the domain. Simulating Physics involves selecting all of the physics models to be used, establishing the region types, the initial and the boundary conditions, and the stopping criteria. Post-Processing is where the results of the simulation are analyzed, both qualitatively and quantitatively.

\subsection{Pre-Processing}

Geometry

Figure 4.1 shows a solid model of the triangular CSELS CapEvap pore geometry. The geometry is created in Solidworks and imported to Star CCM+ as a Parasolid part where it is then used to define the container region. 


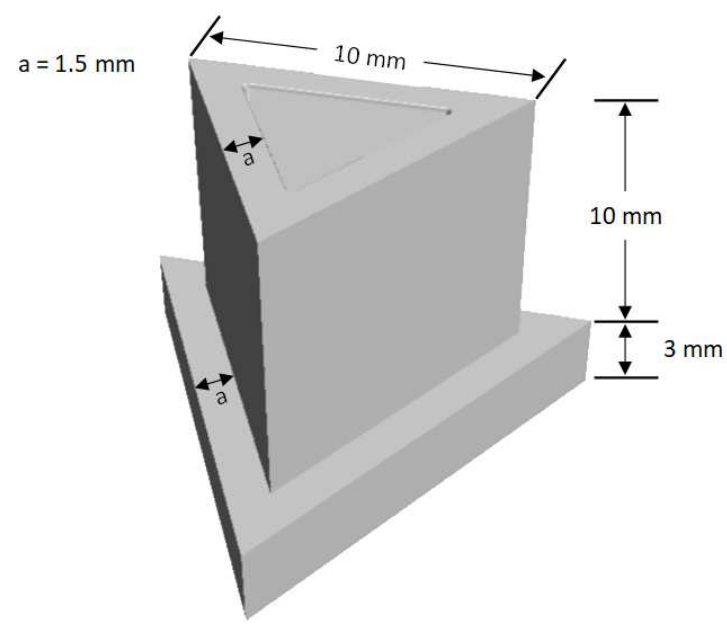

Figure 4.1 - CSELS Triangular Pore - $10 \mathrm{~mm}$ deep with a cross-sectional area of $25 \mathrm{~mm}^{2}$

Region layout

A region is defined in Star $\mathrm{CCM}+$ as a volume, or surface in the case of 2dimensional modelling, completely surrounded on all sides by boundaries. Regions represent the computational domain which is discretized. A multitude of boundary types are available through Star CCM+. However, for this research, boundaries are limited to walls, velocity inlets, and pressure outlets. All models presented herein are comprised of two regions: a multiphase region consisting of air, liquid water, and water vapor, and a solid region consisting of the polycarbonate container or pore structure. The multiphase region has a velocity inlet and a pressure outlet. All other boundaries are walls. Each region shares an interface, highlighted in red in Figure 4.2 allowing for heat transfer between the two regions. 




Figure 4.2 - Regions layout

\section{Meshing}

The mesh is the spatial discretization of the regions in the model. Discretizing the domain allows for the application of the governing equations. Multiple mesh models are available, however, with the polyhedral mesh option enabled, higher quality cells exhibiting a low degree of skewness in the interior corners of the triangular geometry were generated when compared to the cartesian mesh option. Following recommendations made in the user manual, the mesh density is highest at the free surface and prism layers are generated at physical boundaries. Higher mesh density at the free surface allows for more accurate evaporation rates and better mass conservation 
and prism layers increase the accuracy of heat transfer at physical boundaries as shown in Figure 4.4.

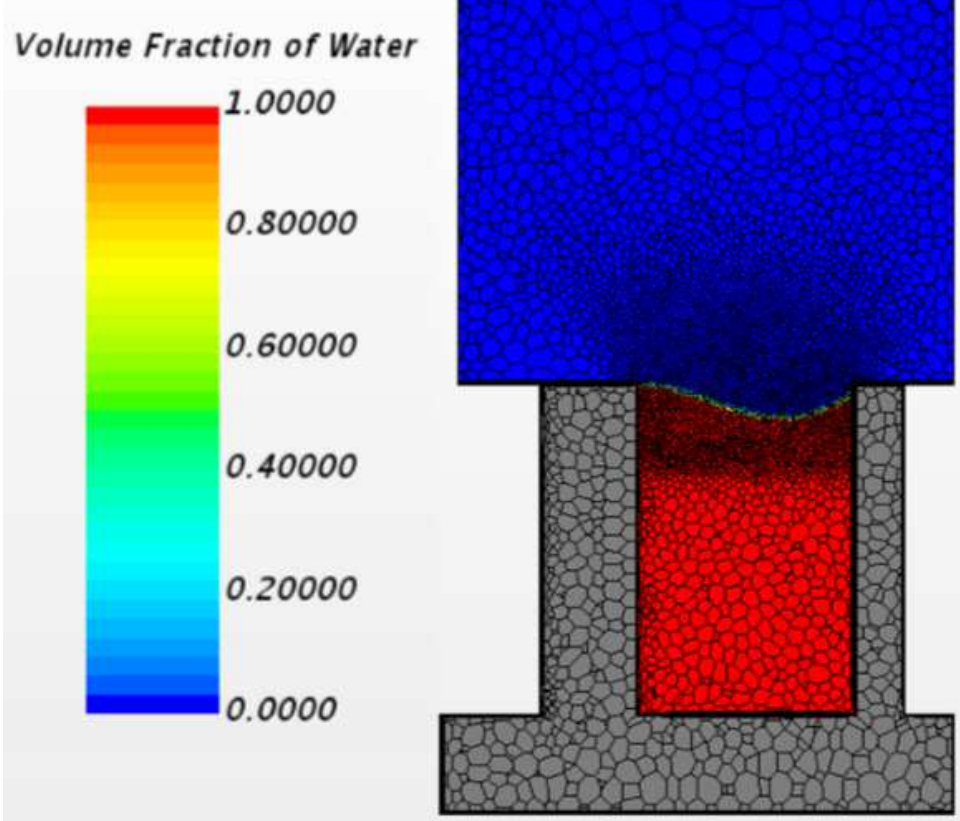

Figure 4.3 - Polyhedral mesh: Triangle Geometry, 95\% fill 


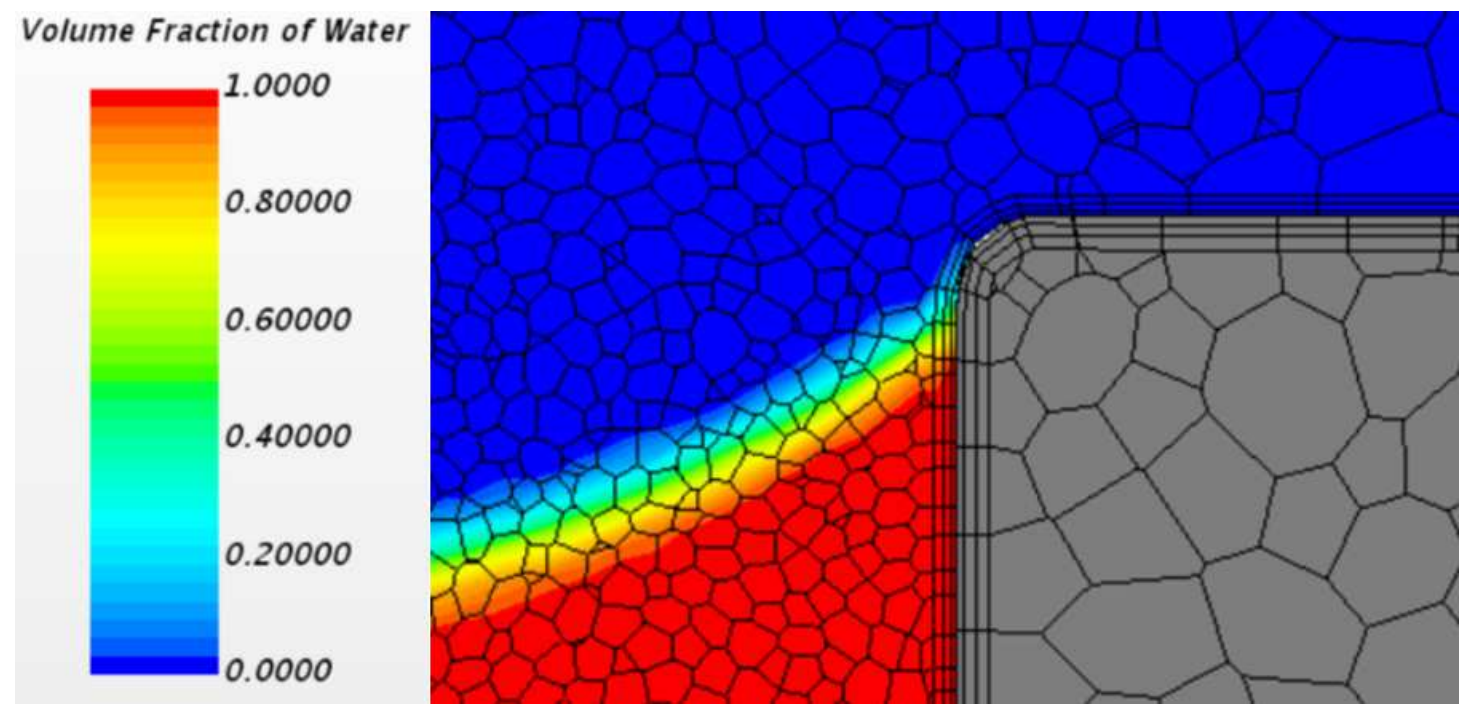

Figure 4.4 - Interface mesh density and Prism layers generated at solid boundaries

\section{2) Simulating Physics}

The following Star $\mathrm{CCM}+$ physics models are employed in all of the simulations herein and will be elaborated further as necessary. The Star CCM+ model selections are capitalized and italicized.

1. Implicit Unsteady

2. Segregated Multiphase Temperature

3. Laminar

4. Gradients

5. Segregated Flow

6. Multiphase Equation of State

a. Water:

i. Constant Density 
b. Air:

i. Ideal Gas in 1-go

ii. Constant Density in microgravity

\section{Eulerian Multiphase}

a. Volume of Fluid for modelling of pores

b. Fluid Film for modelling of foam

\section{Three-dimensional}

Implicit Unsteady

Evaporation is an inherently unsteady process and must be modelled utilizing an unsteady numerical formulation. 'Implicit Unsteady' is recommended when the timescales of the phenomena of interest are the same order as the diffusive process, which is the case for evaporation and therefore is the numerical formulation required by Star $\mathrm{CCM}+$ for modeling evaporation. In contrast to a steady state solver, an unsteady solver requires that a time step be prescribed. The time step must be chosen such that it allows for the accurate capture of the transient phenomena intrinsic to the flow. The criteria used to determine the time step is the Courant-Friedrichs-Lewy (CFL) condition [9]. The CFL condition states that the time step used must satisfy

$$
\Delta t<\frac{\Delta x}{u}
$$


where $\Delta t$ is the time step, $u$ is the maximum velocity in the domain, and $\Delta x$ is the characteristic length of the smallest cell. For the modelling of surface tension dominated flows, the CFL condition is altered to account for the velocity of capillary waves. This stability criteria derived by Brackbill et al. [10] is

$$
\frac{c_{\varnothing} \Delta t_{s}}{\Delta x}<\frac{1}{2}
$$

where $c_{\emptyset}$ is the capillary wave velocity given by

$$
c_{\varnothing}=\left[\frac{\sigma k}{\rho_{1}+\rho_{2}}\right]^{1 / 2} .
$$

Therefore, from equations 4.1 and 4.2 ,

$$
\Delta t_{s}<\left[\frac{<\rho>(\Delta x)^{3}}{2 \pi \sigma}\right]^{1 / 2}
$$

For $\Delta x \approx 10^{-4} \mathrm{~m}$ and the fluid properties of water, we find $\Delta t_{s}<4.7 \times 10^{-5} \mathrm{~s}$.

\section{Physics Continua:}

The following are the appropriate models, or Physics Continua, for use in numerically modelling the CapEvap experiments. As evaporation occurs, a temperature drop results from the latent heat of vaporization of the evaporating liquid which in turn causes temperature changes and heat transfer in the simulation to occur. The Segregated Multiphase Temperature model solves the energy equation (equation 2.8) with temperature as the unknown variable. As the fluid flow in the simulation is laminar, the Laminar Flow Model is selected. CFD requires that gradients of scaler quantities, such as 
Temperature, Pressure, Volume fraction, be calculated. Star CCM+ uses the Hybrid GaussLeast Squares method to accomplish this task. For the Volume of Fluid Method, Star CCM+ requires the Segregated Flow Solver. The Segregated Flow Solver uses the SIMPLE method (Semi-Implicit Method for Pressure Linked Equations) first developed by Spalding and Patankar to numerically solve the Navier-Stokes equations. The algorithm is as follows:

1. Set boundary conditions

2. Compute reconstruction gradients of velocity and pressure

3. Compute the velocity and pressure gradients

4. Solve discretized momentum equation creating intermediate velocity field $\mathbf{v}^{*}$

5. Compute uncorrected mass fluxes at faces $\dot{\boldsymbol{m}}_{\boldsymbol{f}}{ }^{*}$

6. Solve pressure correction equation producing cell values for the pressure correction $\boldsymbol{p}^{\prime}$

7. Update pressure field $\boldsymbol{p}^{\boldsymbol{n}+\boldsymbol{1}}=\boldsymbol{p}^{\boldsymbol{n}}+\boldsymbol{\omega} \boldsymbol{p}^{\prime}$, where $\omega$ is the under-relaxation factor for pressure

8. Update boundary pressure corrections $\boldsymbol{p}_{\boldsymbol{b}}^{\prime}$

9. Correct face mass fluxes $\dot{m}_{f}^{n+1}=\dot{m}_{f}^{*}+m_{f}^{\prime}$

10. Correct cell velocities $\mathbf{v}_{p}^{n+1}=\mathbf{v}_{p}^{*}-\frac{\mathrm{v} \nabla \mathrm{p}^{\prime}}{{\boldsymbol{a}_{\mathrm{v}}^{\prime}}_{\mathrm{v}}^{p}}$, where $\nabla \mathrm{p}^{\prime}$ is the gradient of the pressure corrections, $\boldsymbol{a}_{\mathrm{V}}^{\prime p}$ is the vector of central coefficients for the discretized velocity equation, and $V$ is the cell volume

11. Update density due to pressure changes 


\section{Free all temporary storage}

The equations of state must be prescribed to accurately capture the behavior of the materials in the simulations. For simulations in microgravity, all materials, fluids and solid, are considered to be constant density as buoyant forces are negligible. In 1-go, air is modelled as an Ideal Gas. Interactions between the two immiscible fluids, Surface Tension and Evaporation, are activated as multiphase interactions. Eulerian Multiphase and Volume of Fluid are selected as this method allows for two immiscible fluids to occupy one region simultaneously. For the purpose of evaporation or condensation, water and air phases must be defined as Multi-Component Phases allowing for diffusion of species to occur. All models are three-dimensional.

Materials

All thermophysical properties for each material must be prescribed. The properties of liquid water, water vapor, air, and Polycarbonate used are listed in Table 4.1. The material properties listed in Table 4.1 are for pure substances. As species diffusion occurs new properties must be calculated as either mass weighted or volume weighted where Density is volume-weighted, Dynamic Viscosity, Specific Heat, and Thermal Conductivity are massweighted. 
Table 4.3 - Boundary Conditions

\begin{tabular}{|c|c|}
\hline \multicolumn{2}{|r|}{ Boundary Conditions: } \\
\hline Inlet & $\begin{array}{l}\text { Velocity }=0.05 \mathrm{~m} / \mathrm{s} \\
\text { Temperature: } 22^{\circ} \mathrm{C} \\
\text { Mass Fraction: } \\
\quad \text { Air: } 0.9935 \\
\circ \quad \text { Water: } 0.0065\end{array}$ \\
\hline Outlet & $\begin{array}{l}\text { Pressure }=101.325 \mathrm{kPa} \\
\text { Temperature: } 22^{\circ} \mathrm{C} \\
\text { Mass Fraction: } \\
\circ \quad \text { Air: } 0.9935 \\
\circ \quad \text { Water: } 0.0065\end{array}$ \\
\hline Container Exterior & $\begin{array}{l}\text { Convection Boundary Condition } \\
\qquad \quad \text { Temperature: } 295.15 \mathrm{~K} \\
\circ \quad \text { Convection coefficient: } 1.41 \frac{\mathrm{W}}{\mathrm{m}^{2} \mathrm{~K}} \\
\text { All other physical boundaries are adiabatic walls. }\end{array}$ \\
\hline Interfaces & $\begin{array}{l}\text { Contact angle: } 48^{\circ} \\
\text { Conjugate heat transfer }\end{array}$ \\
\hline
\end{tabular}

Table 4.4 - Stopping Criteria

\begin{tabular}{|l|ll|}
\hline \multicolumn{2}{|c|}{ Stopping Criteria } \\
\hline Residuals: & $\bullet$ & Energy: 1E-4 \\
& $\bullet$ & Continuity: 1E-4 \\
& $\bullet$ & X, Y, and Z momentum: 1E-4 \\
& $\bullet$ & Water: 1E-4 \\
& & \\
\hline Evaporation Rate & Quasi-steady \\
\hline
\end{tabular}




\subsection{Post-Processing}

Post processing is accomplished with both Scalar Scenes and plots. Scalar Scenes are a qualitative measure of a scalar of interest such as a temperature, pressure, humidity and more. Plots are quantitative in nature and are valuable in assessing time dependent processes. Specifically, plots are used herein to determine when processes such as evaporation have achieved a quasi-steady. 


\section{Chapter 5}

\section{Benchmark}

The VOF evaporation model in Star CCM+ is benchmarked against the analytical solution to the Stefan tube outlined in chapter 4. A schematic of the Stephan tube model is shown in Figure 5.1. Multiple fill levels are chosen and run using the code until the evaporation rate reaches a quasi-steady state. By multiplying equation 3.30 by the free surface area we formulate an analytical solution to the evaporation rate in $\mathrm{kg} / \mathrm{s}$ as a function of the fill level in a Stefan tube: namely,

$$
\frac{d Z}{d t}=\left(\frac{D_{A B} P M_{A}\left(P_{A 1}-P_{A 2}\right)}{2 t \rho_{A, L} P_{B, l m} R T}\right)^{1 / 2}\left(\rho_{A, L} A_{S}\right) .
$$

In equation $5.1, A_{S}$ is the surface area and $\rho_{A, L}$ is the density of the evaporating liquid. 


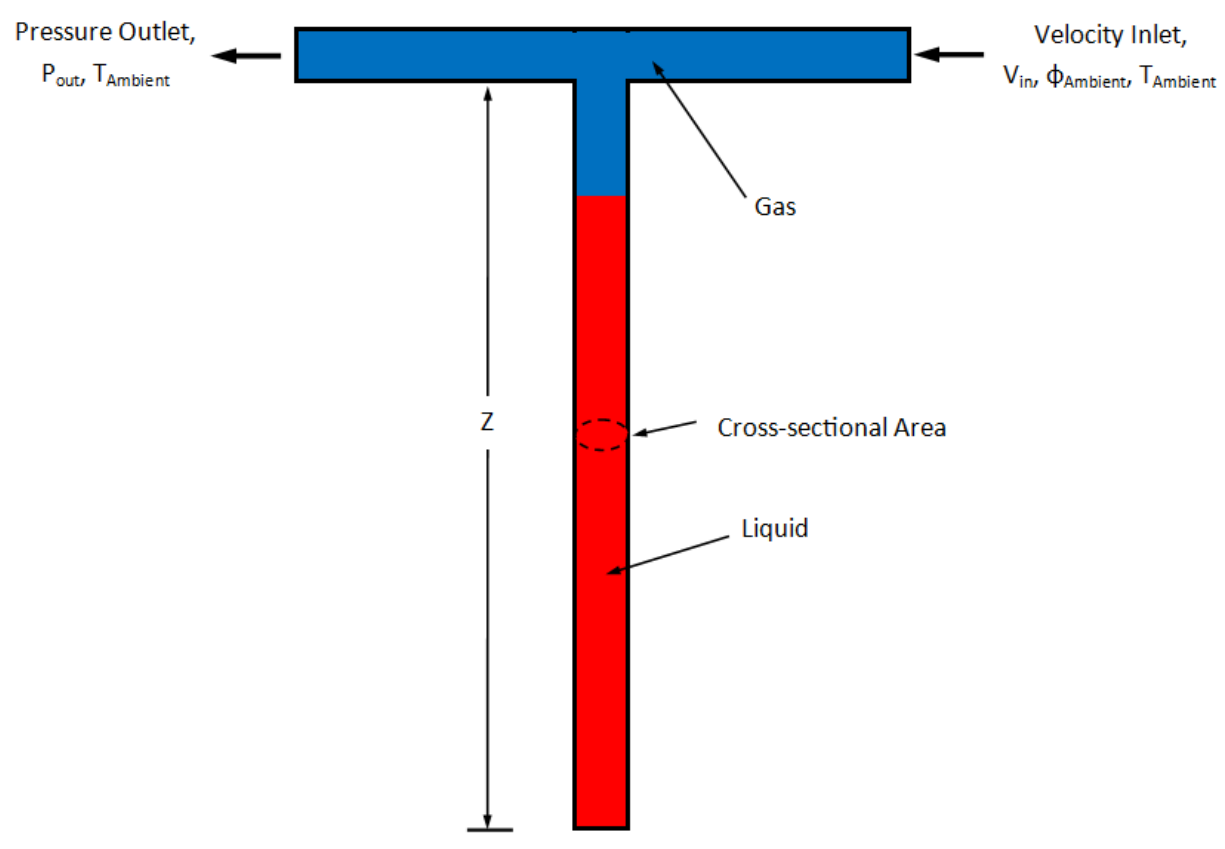

Figure 5.1 - Stefan Tube: $Z=50 \mathrm{~mm}$, Fill levels $=25 \mathrm{~mm}, 31.25 \mathrm{~mm}, 37.5 \mathrm{~mm}, 43.75 \mathrm{~mm}, A c=7.854 \times$ $10^{-7} \mathrm{~m}^{2}, V_{\text {in }}=1 \mathrm{~m} / \mathrm{s}, P_{\text {out }}=1$ atmosphere



Figure 5.2 - Fill level vs. Evaporation rate for equation 5.1 and the Volume of Fluid evaporation model 
Table 5.1 - Fill level vs. Evaporation rate for the Analytical solution and the Volume of Fluid model

\begin{tabular}{|l|l|l|l|}
\hline $\begin{array}{c}\mathrm{C} \\
(\mathrm{mm})\end{array}$ & \multicolumn{1}{|c|}{$\begin{array}{c}\text { Analytical Evaporation } \\
\text { Rate }(\mathrm{kg} / \mathrm{s})\end{array}$} & \multicolumn{1}{c|}{$\begin{array}{c}\text { Volume of Fluid Evaporation Rate } \\
(\mathrm{kg} / \mathrm{s})\end{array}$} & \multicolumn{1}{c|}{ Percent Difference } \\
\hline 43.75 & $3.81 \mathrm{E}-11$ & $3.86 \mathrm{E}-11$ & $+1.2 \%$ \\
\hline 37.5 & $1.92 \mathrm{E}-11$ & $1.92 \mathrm{E}-11$ & $-0.4 \%$ \\
\hline 31.25 & $1.28 \mathrm{E}-11$ & $1.27 \mathrm{E}-11$ & $-1.1 \%$ \\
\hline 25 & $9.62 \mathrm{E}-12$ & $9.50 \mathrm{E}-12$ & $-1.2 \%$ \\
\hline
\end{tabular}

From Figure 5.2 and Table 5.1 it is observed that the Star CCM+ VOF method accurately predicts the analytical solution to within an average percent difference of $\pm 1 \%$. At high fill levels the model slightly over-predicts and as the fluid interface recedes into the tube the model slightly under-predicts. 


\section{Chapter 6}

\section{Results}

The results presented in this section are analyzed to investigate the efficacy of Star $\mathrm{CCM}+$ to model multiphase flows with evaporative phase change in a microgravity environment, specifically those carried out by NASA in the CSELS CapEvap experiments on ISS. We benchmarked the code to an idealized Stefan Tube analytical solution in Chapter 5. As a first assessment, we then compare evaporation rates predicted by the numerical models with those measured during the CapEvap experiments. The results of the models are then used to investigate the contributions of the evaporating surface area and contact line length to the total evaporation rates. The effects of gravity are investigated in a manner to isolate the mechanism responsible for the difference in evaporation rates observed experimentally between terrestrial and microgravity environments. Lastly, weighted Stefan Tube equations are compared to the experimental results to establish a quantitative method with which to predict evaporation aboard 
spacecraft. As an application of the numerical model, the thermal problem of the CapEvap multi-pore test cell is solved which illuminates otherwise bewildering experimental results observed.

\subsection{Numerical and Experimental Comparisons}

Figure 6.1 shows the evaporation rates measured during the CapEvap experiments. As can be seen, in each plot there are two regions of approximately linear evaporation rates. Two pore fill volumes are chosen corresponding to the two regions of $95 \%$ and $75 \%$. Table 6.1 compares the evaporation rates from Star CCM+ for the two fill rates with the evaporation rates of the linear regions in the Figure.

Table 6.1 - Star CCM+ Evaporation rates, Square and Triangle, 95\% fill and 75\% fill

\begin{tabular}{|c|c|c|c|}
\hline Fill Percentage & $\begin{array}{c}\text { CapEvap Evaporation Rate } \\
(\mathrm{kg} / \mathrm{s})\end{array}$ & $\begin{array}{c}\text { Star CCM+ Evaporation Rate } \\
(\mathrm{kg} / \mathrm{s})\end{array}$ & Percent Difference \\
\hline \multicolumn{3}{|c|}{ Square Geometry } \\
\hline 0.95 & $4.65 \mathrm{E}-09$ & $4.23 \mathrm{E}-09$ & $-10 \%$ \\
\hline 0.75 & $1.90 \mathrm{E}-09$ & $2.18 \mathrm{E}-09$ & $+14 \%$ \\
\hline \multicolumn{5}{|r|}{ Triangle Geometry } \\
\hline 0.95 & $4.07 \mathrm{E}-09$ & $4.13 \mathrm{E}-09$ & $+1 \%$ \\
\hline 0.75 & $2.71 \mathrm{E}-09$ & $2.38 \mathrm{E}-09$ & $+13 \%$ \\
\hline
\end{tabular}




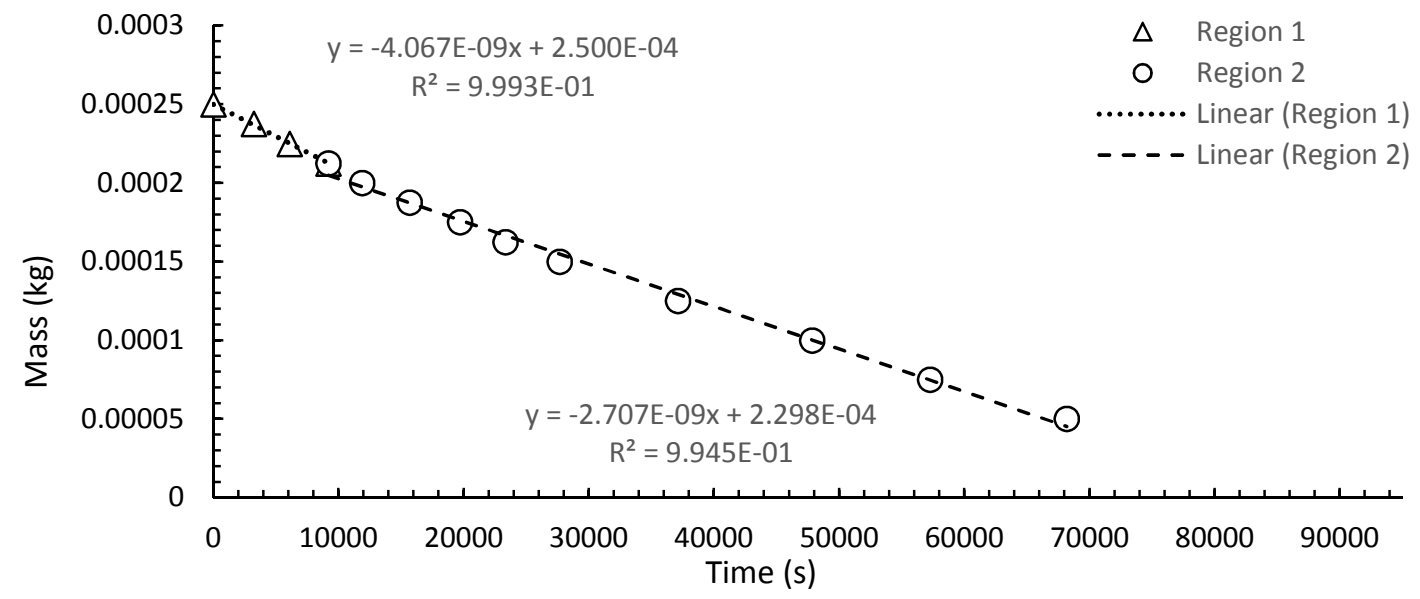

(a)

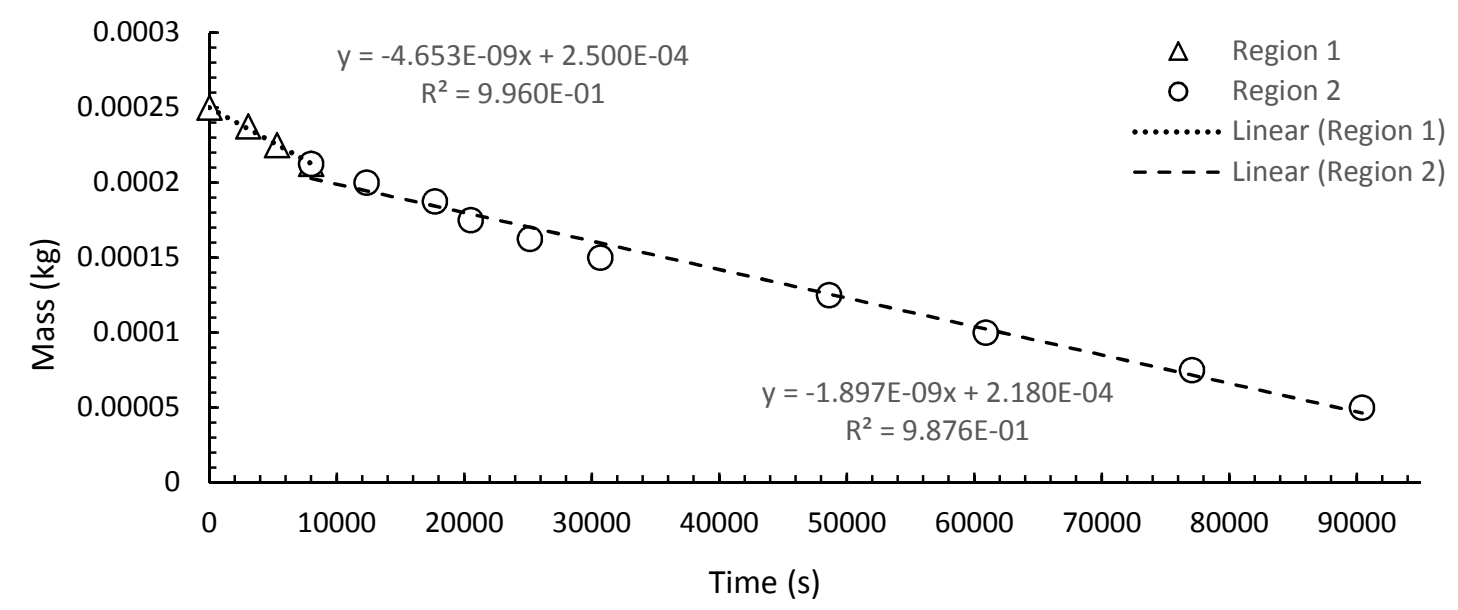

(b)

Figure 6.1 - Experimental results: Mass of water $(\mathrm{kg})$ as a function time $(\mathrm{s})$ for (a) triangular pore geometry and (b) square pore geometry

From Table 6.1 it is seen that Star CCM+ reproduces experimental evaporation rates to within a maximum percent difference of approximately $\pm 14 \%$. While the interior corners of the triangular geometry remain wetted throughout the evaporation process, SE-FIT shows that the interior corners of the square geometry remain wetted only until between $15 \%$ and $20 \%$ of the volume has evaporated. This may explain why the square exhibits a 
higher evaporation rate in the first linear region when compared with the triangular geometry, both in the numerics and experiments, as the square establishes more free surface area at the entrance to the pore.

\subsection{Surface area vs. contact line length}

Evaporation occurs at the free surface between a liquid and vapor phase and as such increasing the surface area will increase the evaporation rate. However, when the fluid is in contact with a boundary that can supply heat, as with the 'pores' of the CapEvap experiments, we expect an increase in evaporation at the boundaries due to the local increased heat flux to the free surface. Figure 6.2 (a) shows the local evaporation rate along section A-A of the free surface within the triangle pore with a fill level of $75 \%$. As expected, we compute an increased evaporation rate at the contact line regions, with an approximate three-fold increase in local evaporation rate at the interior corner (left). 


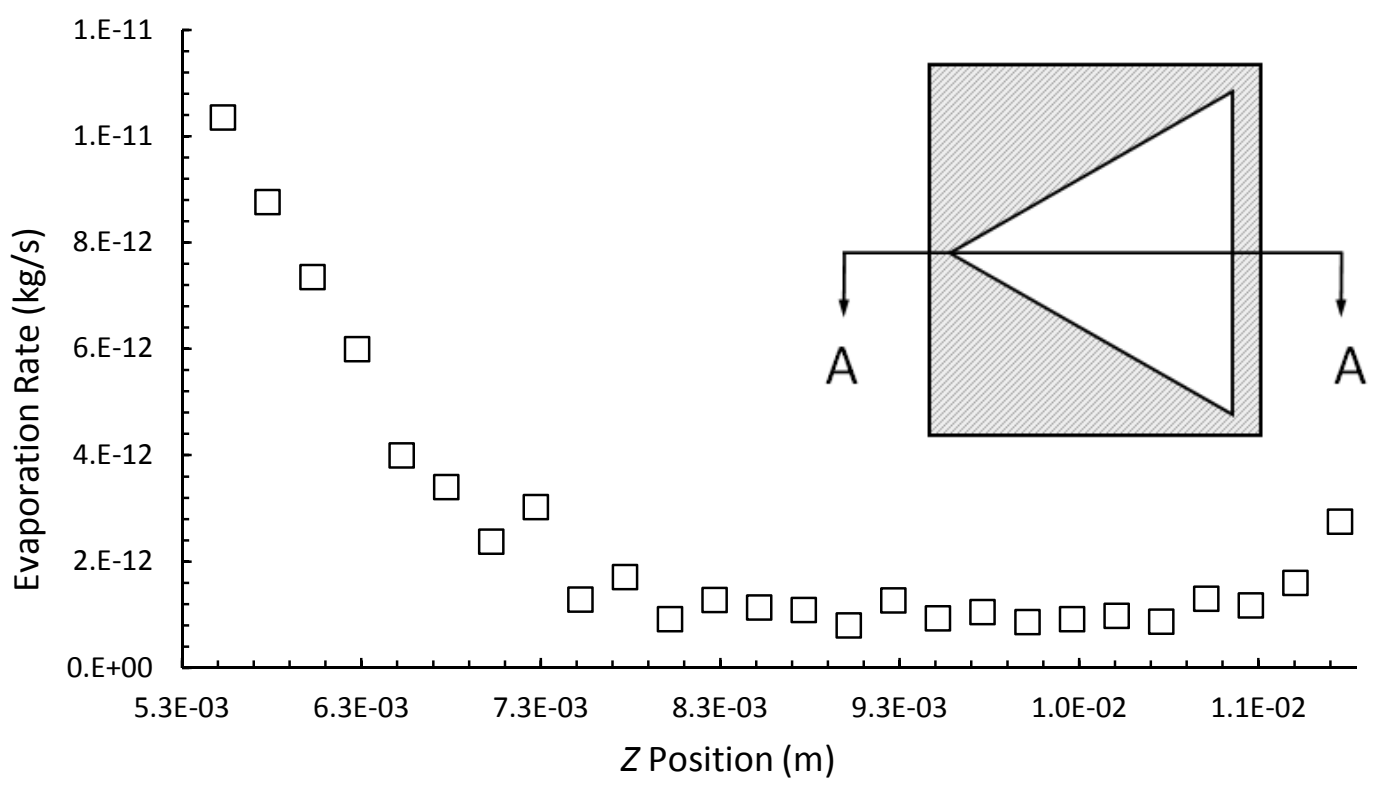

(a)
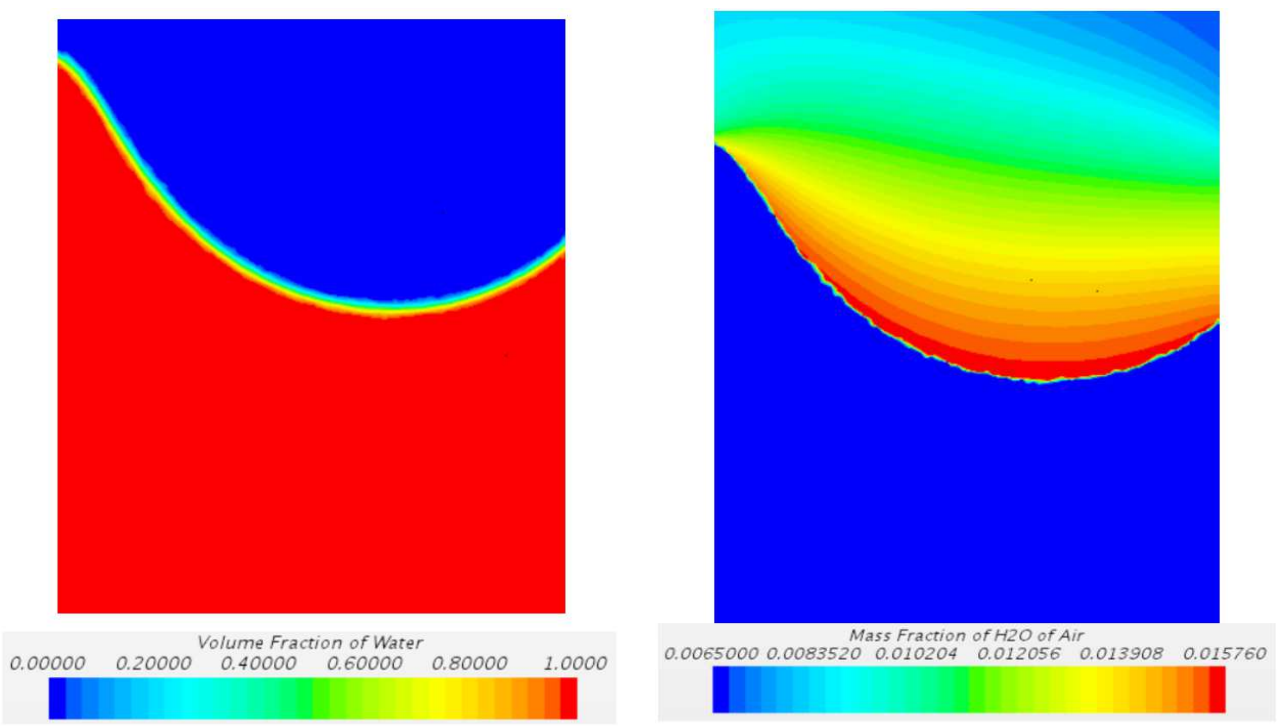

(b)

(c)

Figure 6.2 - (a): Evaporation rate along section A-A in triangular geometry with a 75\% fill fraction, (b): Cross-sectional view showing volume of fraction of water from triangular pore with a $75 \%$ fill, (c): Cross-sectional view showing mass fraction of water above free surface from triangular pore with a 75 percent fill 
Due to the wetting characteristics and the triangular geometry, the receding liquid interface wets the interior corner while outside of the interior corner region the liquid interface recedes into the pore during evaporation. This is the result of the receding contact angle satisfying the Concus-Finn condition for the corner; namely $\theta_{\text {rec }}<\frac{\pi}{2}-\alpha=$ $60^{\circ}$ [8]. Figure 6.2 (b) shows both the minimum and maximum vertical position of the contact line region in the triangular pore. It is believed that this corner wetting is the primary mechanism driving the large difference in evaporation rate the contact line region in the corners - the rivulets along the corner provide a capillary pumping mechanism to drive liquid into regions of lower local saturation (humidity) and thus enhance evaporation. The Stefan Tube solution suggests that the time for the free surface to recede from position $Z_{1}$ to $Z_{2}$ is proportional to the difference of the square of the positions. In other words the evaporation rate slows as the free surface recedes into the pore as $t \sim Z_{t}^{2}$ which is in agreement with Figure 6.2 as the boundary with the highest evaporation rate occurs where the fluid interface is pinned flat at the opening of the pore. Figure 6.2 (c) shows the humidity gradient above the free surface which is highest for the elevated liquid column in the interior corners. As can be seen, the evaporation is highest at the surface exposed to the lowest relative humidity which is at the top near the interior corners. 
To isolate the relative contribution of the contact line region to the overall evaporation rate, the free surface must have a constant height for a given pore. Figure 6.3 (a) shows the evaporation rate along section A-A as with Figure 6.2 (a), but, with a fill level of $100 \%$. With the entire flat free surface at a constant height we observe the contribution of the contact line region only. There is slight asymmetry in evaporation rates between the two sides of the container which is understood by looking at the asymmetric mass fraction of water vapor in Figure 6.3 (b). Due to the airflow over the free surface, an asymmetric humidity boundary layer is formed. This boundary layer creates only a slight asymmetry in the humidity gradient above the free surface which in turn is responsible for the slight asymmetry in observed evaporation rates. Figure 6.4 shows the evaporations rates over the entire free surface for both the (a) square and (b) triangular CSELS CapEvap pore geometries with a 75\% fill level. As can be seen, the evaporation rates in the interior corners of the triangular geometry are approximately three times greater than those of the square geometry. As the interface further recedes into the pore, this difference will grow, as is seen in the results from the CapEvap experiments, as evaporation rates are a function of the height of the free surface. 


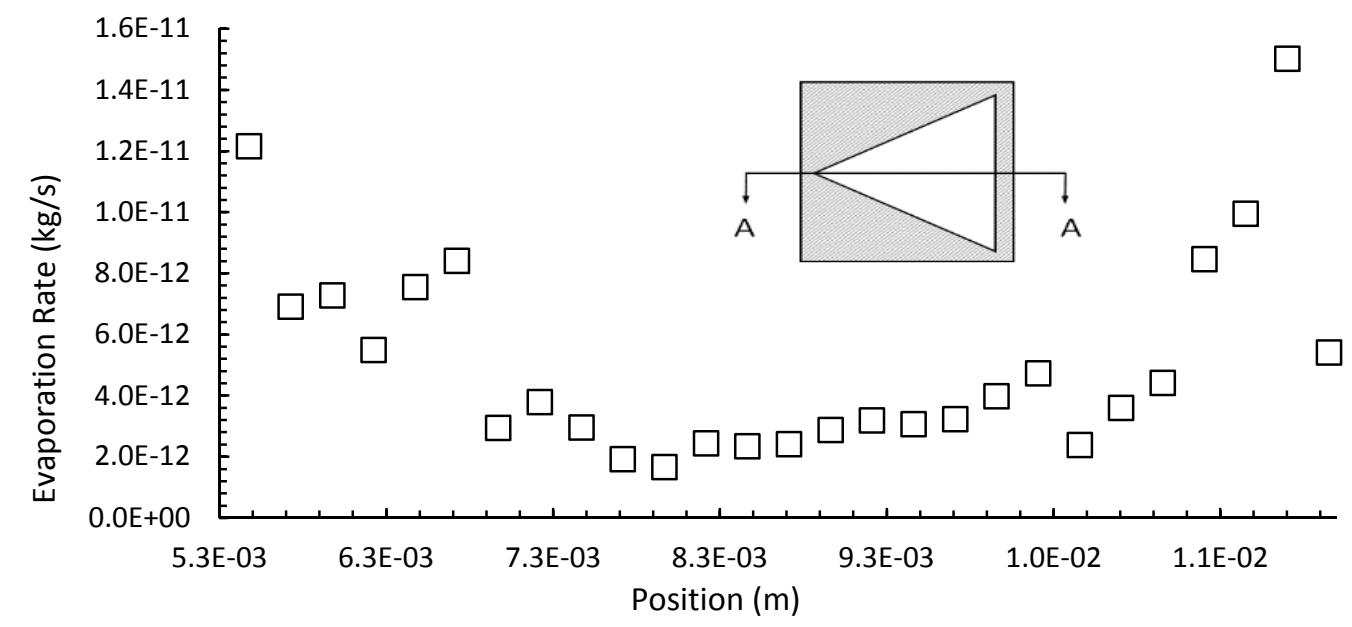

(a)



(b)

Figure 6.3 - (a): Evaporation rate along Section A-A, (b): Boundary layer over CapEvap triangular geometry, 100\% fill level

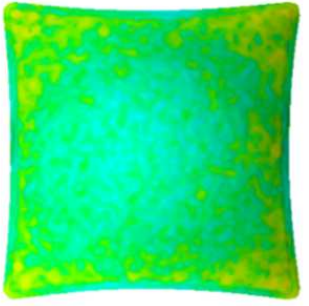

(a)

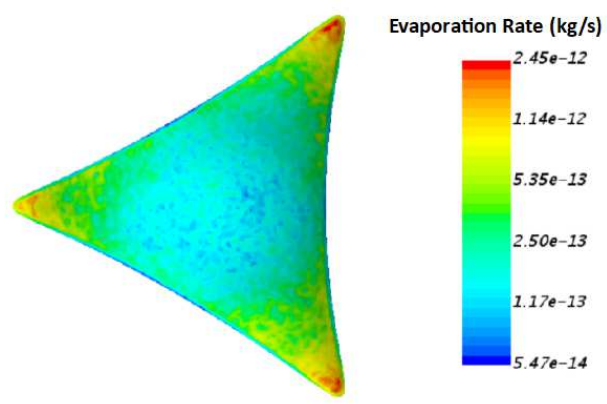

(b)

Figure 6.4 - Numerical Evaporation rate of free surface: (a) CapEvap Square Geometry, 75\% fill level. (b) CapEvap Triangle Geometry, $75 \%$ fill level. Parallax due to perspective 


\subsection{Terrestrial and reduced gravity environments}

Based on the CSELS CapEvap experiments, in nearly identical surroundings ( $T=$ 295.1K, $P=101.3 \mathrm{kPa}, \phi=40 \%$ ), evaporation rates in $1-g_{0}$ were observed to be higher than those in microgravity by as much as $40 \%$ at low airflow velocity. It has been assumed that buoyancy in the vapor phase is responsible for the majority of the difference. The results from the CFD models comparing 1-go evaporation rates with those in microgravity are given in Table 6.2. From the table, the rate of evaporation between 1$g_{0}$ and microgravity varies between $15 \%$ and $60 \%$ with the microgravity evaporation showing a strong dependence on airflow velocity. As the airflow velocity increases, the effects of gravity are diminished as forced convection increases in importance. The model also shows a relatively uniform temperature (Figure 6.5) at the surface of the foam block 286.6 $\mathrm{K} \leq T \leq 287.0 \mathrm{~K}$. When solving the heat transfer problem, the Richardson number $R i=g \beta\left(T_{s}-T_{\infty}\right) L_{x}^{3} /\left(\rho u L_{y} / \mu\right)^{2}$, where $L_{x}$ is along the direction of forced air flow and $L_{y}$ is the direction in which gravity acts, can be thought of as the ratio of the Reynolds numbers for natural and forced convection and is employed to determine if forced or natural convection dominate thermal convection. Figure 6.6 provides a semilog plot of Ri calculated for the geometry of the CapEvap foam block given in Figure 1.1 (a) as a function of the air velocity. The plot reveals that for an airflow velocity of $5 \mathrm{~cm} / \mathrm{s}$ in $1-g_{0}$, natural convection may be neglected. However, for an airflow velocity of $1 \mathrm{~cm} / \mathrm{s}$ in 1-go neither natural or forced convection may be neglected. This situation is borne out in Table 6.2 where in $1-g_{0}$ the evaporation rate at $5 \mathrm{~cm} / \mathrm{s}$ is 1.04 times that of $1 \mathrm{~cm} / \mathrm{s}$, 
whereas in microgravity, $5 \mathrm{~cm} / \mathrm{s}$ yields an evaporation rate 1.6 times higher than that of $1 \mathrm{~cm} / \mathrm{s}$.

Table 6.2 - CSELS Foam: 1-govs. Microgravity evaporation rates

\begin{tabular}{|c|c|c|c|}
\hline \multicolumn{3}{|c|}{ CFD Results: Foam } \\
\hline Velocity $(\mathrm{m} / \mathrm{s})$ & $\begin{array}{c}1-g_{0} \text { Evaporation } \\
\text { Rate }\left(\mathrm{g} / \mathrm{m}^{2} \mathrm{hr}\right)\end{array}$ & $\begin{array}{c}\text { Microgravity } \\
\text { Evaporation Rate }\left(\mathrm{g} / \mathrm{m}^{2} \mathrm{hr}\right)\end{array}$ & $\begin{array}{c}\text { Percent } \\
\text { Difference }\end{array}$ \\
\hline 0.10 & 89.28 & 84.96 & $5 \%$ \\
\hline 0.05 & 78.34 & 65.09 & $18 \%$ \\
\hline 0.01 & 75.60 & 40.43 & $61 \%$ \\
\hline
\end{tabular}

Table 6.3 - CapEvap CSELS Foam block evaporation temperatures: Min, Max, Mean, V=1 cm/s, and $5 \mathrm{~cm} / \mathrm{s}$ in 1-go, and microgravity

\begin{tabular}{|c|r|r|r|r|r|r|}
\cline { 2 - 7 } \multicolumn{1}{c|}{} & \multicolumn{5}{|c|}{ Temperature (K) } \\
\cline { 2 - 7 } \multicolumn{1}{c|}{} & \multicolumn{4}{|c|}{ Microgravity } & \multicolumn{1}{c|}{$1-g_{0}$} \\
\hline Velocity (cm/s) & \multicolumn{1}{c|}{ Min } & \multicolumn{1}{c|}{ Max } & Mean & Min & Max & Mean \\
\hline 1 & 286.96 & 287.03 & 287.02 & 286.59 & 286.76 & 286.69 \\
\hline 5 & 286.76 & 286.99 & 286.92 & 286.58 & 286.79 & 286.69 \\
\hline
\end{tabular}

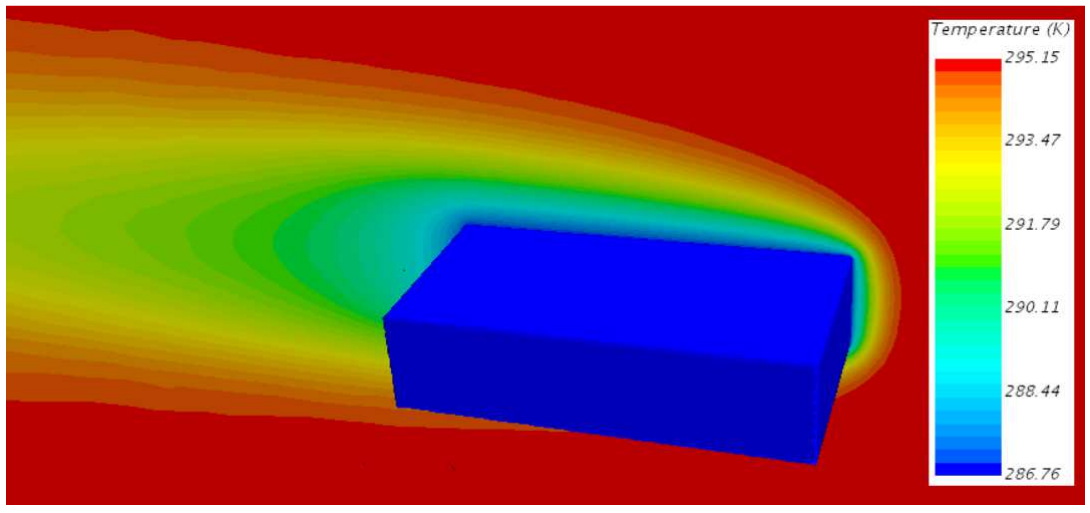

Figure 6.5 - Surface temperature of CapEvap CSELS Foam during evaporation, $V=5 \mathrm{~cm} / \mathrm{s}, g=10^{-6} \mathrm{~g}_{0}$ 


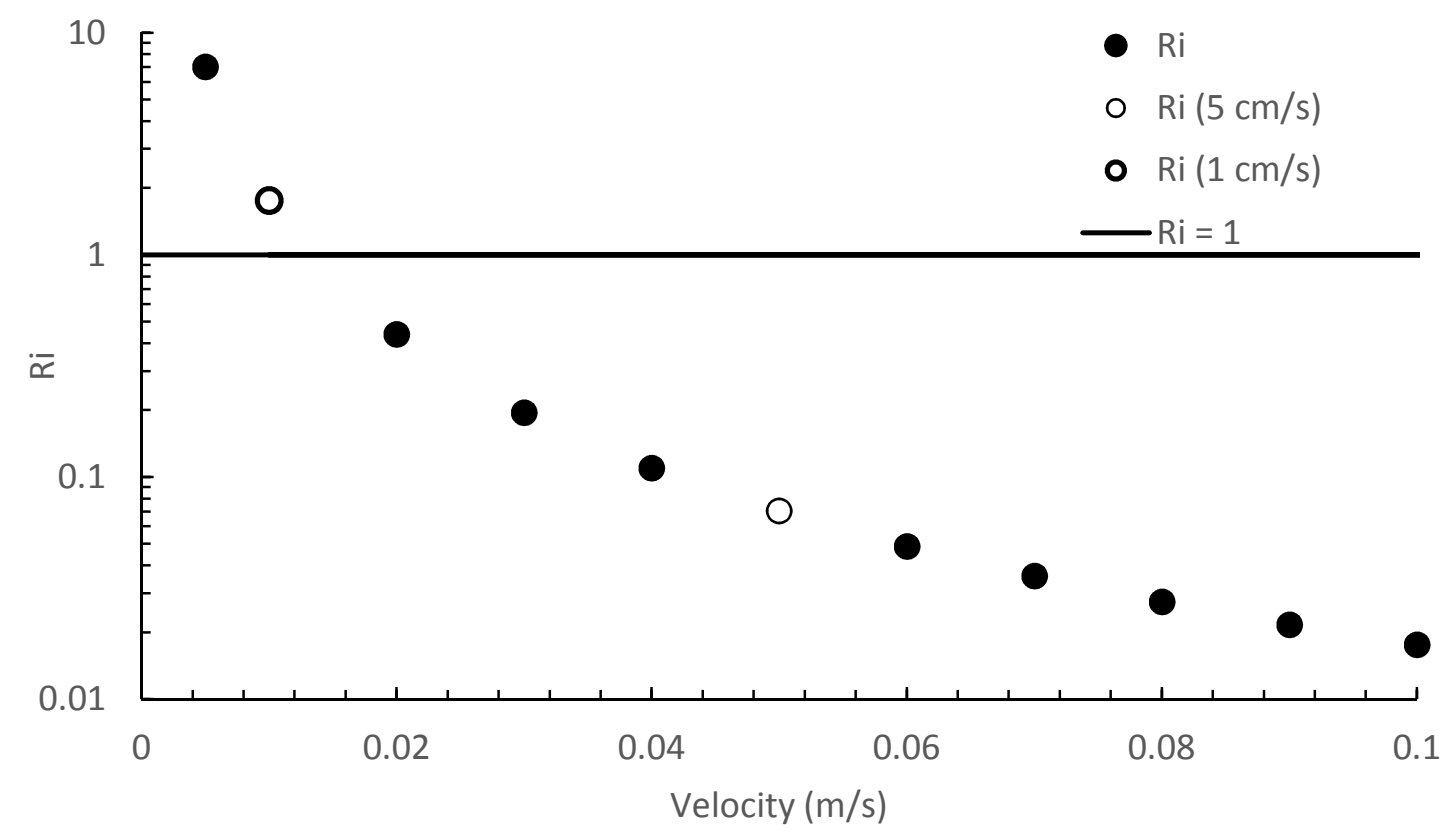

Figure 6.6 - Semi-Log plot of Ri vs. airflow velocity for CapEvap Foam Geometry

\section{4) Weighted Stefan Tube}

The experimental data for the free surface as a position of time as well as Equations 3.29 (Stefan tube), 3.31 (Area weighted Stefan tube), 3.32 (Contact line weighted Stefan tube) are plotted in Figure 6.7 for both the CapEvap square (a) and triangle (b) pores. The Stefan tube equation initially predicts the data well. However, as the free surfaces recede into their respective pores all equations over-predict the height of the free surface and thus under-predict the evaporation rate. Both area (Eq. 3.31) and contact line (Eq. 3.32) weighting are seen to significantly improve the accuracy of the results with the contact line weighting providing the greatest accuracy (Table 6.4). Figure 6.8 displays the linear 
regions of Figure 6.7 where the slopes represent the evaporation rates for the square (a) and triangle (b). In this region Eq 3.32 predicts the evaporation rate to within $6.1 \%$ for the triangle and within $4.4 \%$ for the square (ref. Table 6.5).

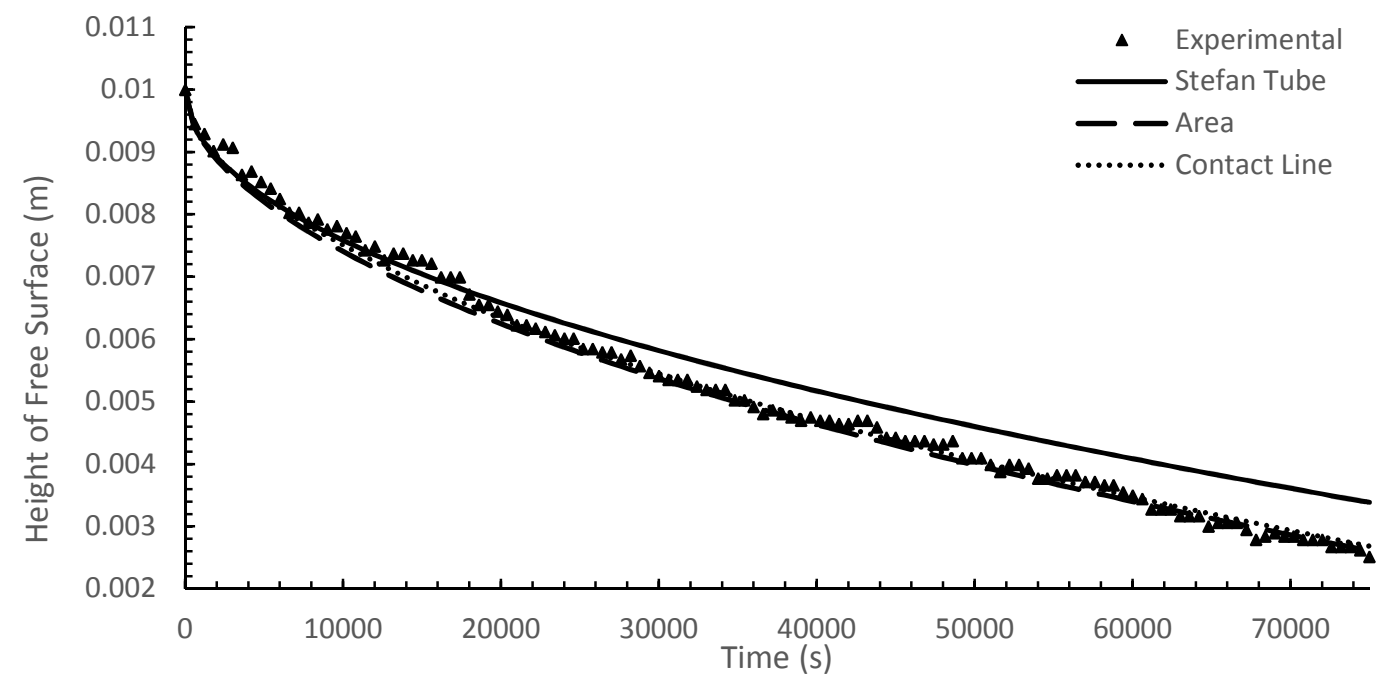

(a)

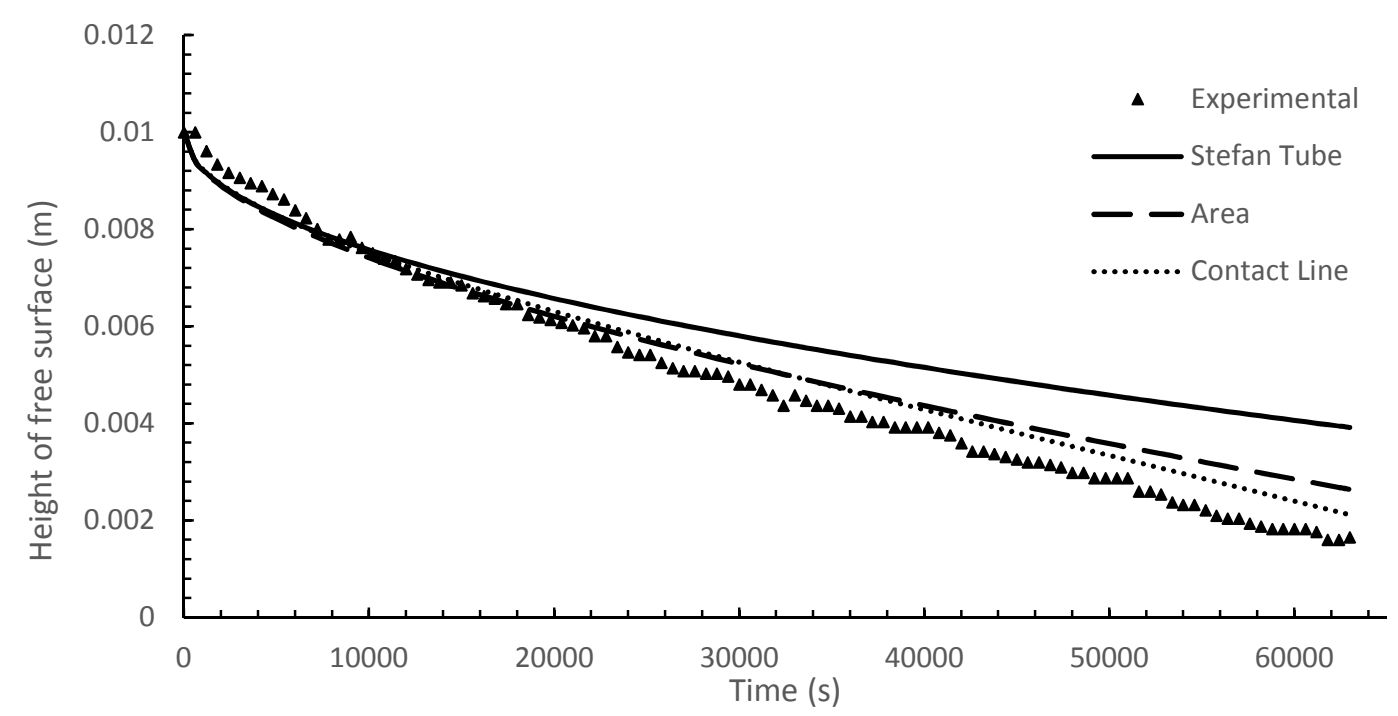

(b)

Figure 6.7 - Position of free surface as a function of time compared with equations 3.29, 3.31 and 3.32 for (a): Square pore geometry, and (b): Triangular pore geometry 
Table 6.4 - Stefan Tube weighted by Area and Contact line

\begin{tabular}{|l|r|l|r|}
\hline \multicolumn{2}{|c|}{ Area } & \multicolumn{2}{c|}{ Contact Line } \\
\hline \multicolumn{2}{|c|}{ Square } \\
\hline Average & $11 \%$ & Average & $23 \%$ \\
\hline Maximum & $26 \%$ & Maximum & $7 \%$ \\
\hline Standard Deviation & $7 \%$ & Standard Deviation & $11 \%$ \\
\hline & \multicolumn{1}{|c|}{ Triangle } & $33 \%$ \\
\hline Average & $14 \%$ & Average & $9 \%$ \\
\hline Maximum & $52 \%$ & Maximum & \\
\hline Standard Deviation & $14 \%$ & Standard Deviation & \\
\hline
\end{tabular}

Table 6.5 - Slope of experimental data and Equation 3.32 in linear region (Figure 6.8)

\begin{tabular}{|l|r|r|r|}
\cline { 2 - 4 } \multicolumn{1}{c|}{} & \multicolumn{3}{c|}{ Evaporation Rate $\left(\mathrm{kg} / \mathrm{m}^{2} \mathrm{~s}\right)$} \\
\cline { 2 - 4 } \multicolumn{1}{c|}{} & Experimental Data & Equation 3.32 & Percent Difference \\
\hline Triangle & $-9.60 \mathrm{E}-08$ & $-1.02 \mathrm{E}-08$ & 6.1 \\
\hline Square & $-6.68 \mathrm{E}-89$ & $-6.39 \mathrm{E}-08$ & 4.4 \\
\hline
\end{tabular}




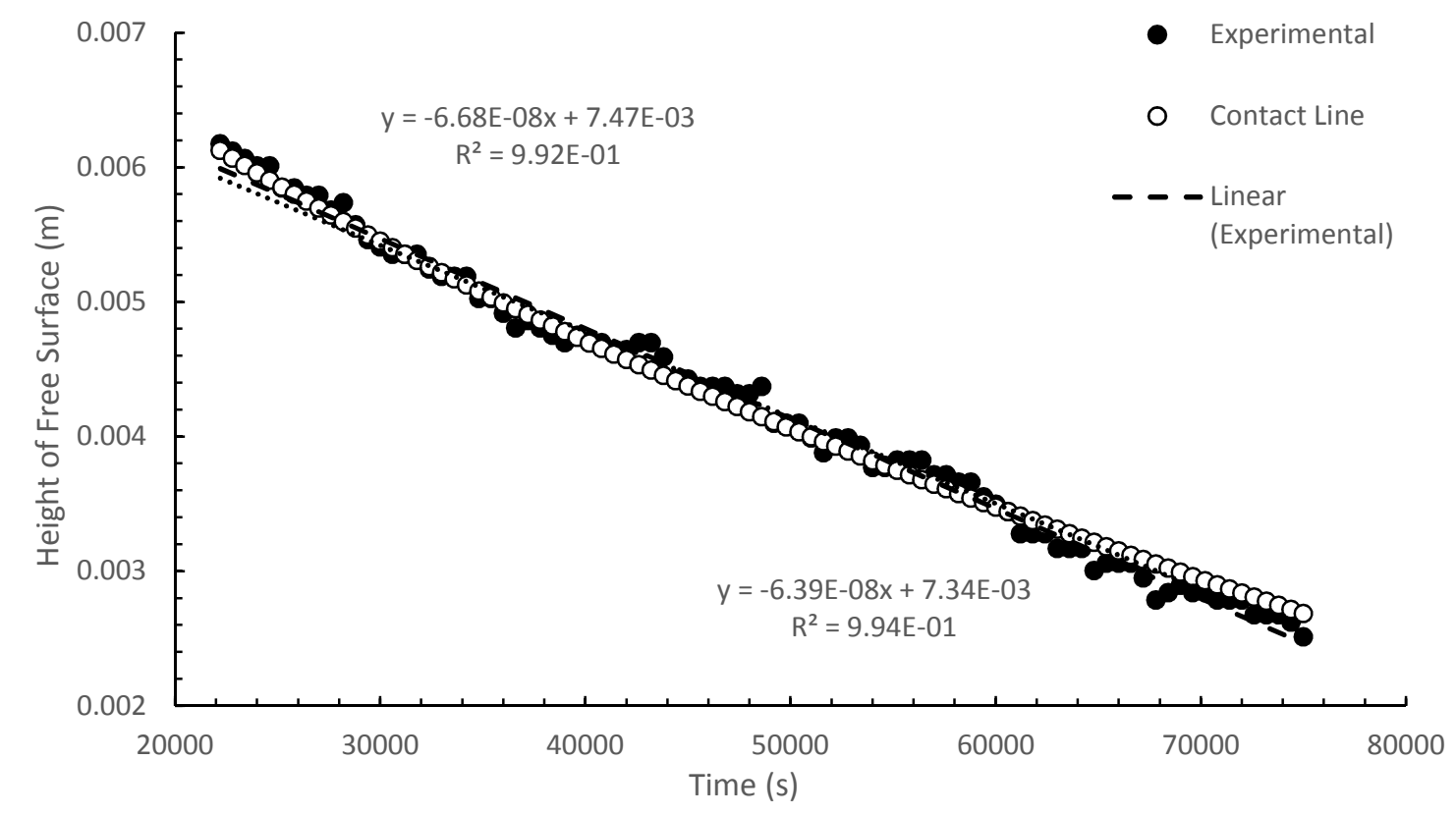

(a)

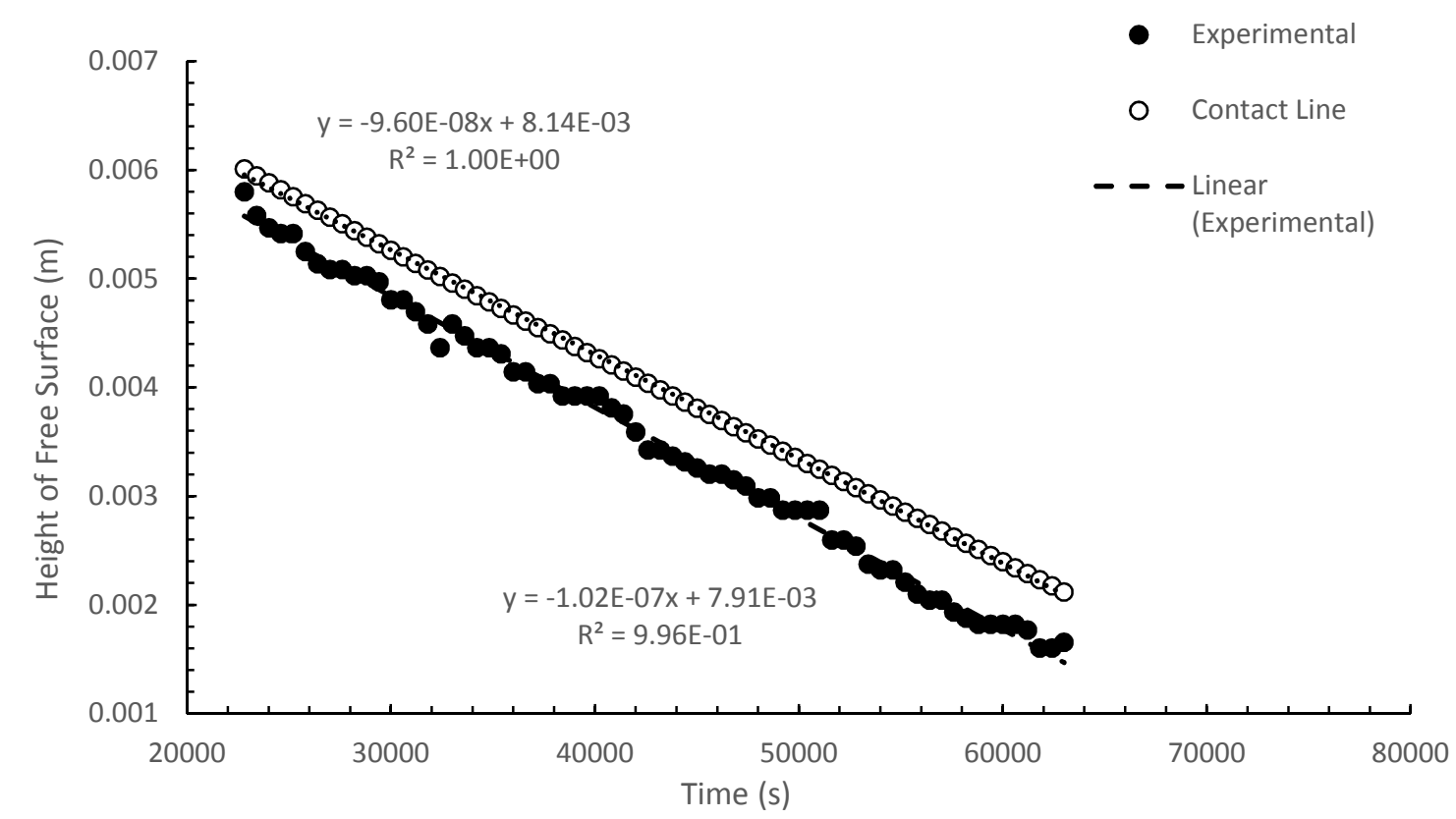

(b)

Figure 6.8 - Linear region evaporation: Experimental data vs Equation 3.32 for (a) square pore geometry, and (b) triangular pore geometry 


\section{Application}

The numerical analyses provided to this point portrays the CSELS pores as individual pores thermally isolated from one another. The actual CSELS pores are grouped together as depicted in Figure 6.9 forming a more complex thermal system. In this demonstration of the Star $\mathrm{CCM}+$ model, the impact of the grouping of pores are investigated using a heat transfer model to identify the impacts, if any, of grouped cells to the evaporation rates. Figure 6.10 (a) shows the heat flux to the free surface of each pore. Asymmetries in heat flux are immediately noticeable. The pore walls exposed to ambient air conditions contribute more heat than do those walls in between the adjacent pores providing a degree of insulation. Thus, we observed reflective symmetry between Pores 1 and 3, and 4 and 5. Further, we note that Pore 1 experiences a greater heat flux than does pore 3, a subtle asymmetry due to a fun effect of the single-piece 3-D printed pore support base that functions as a heat source affecting the temperature, most significantly to Pore 1. 


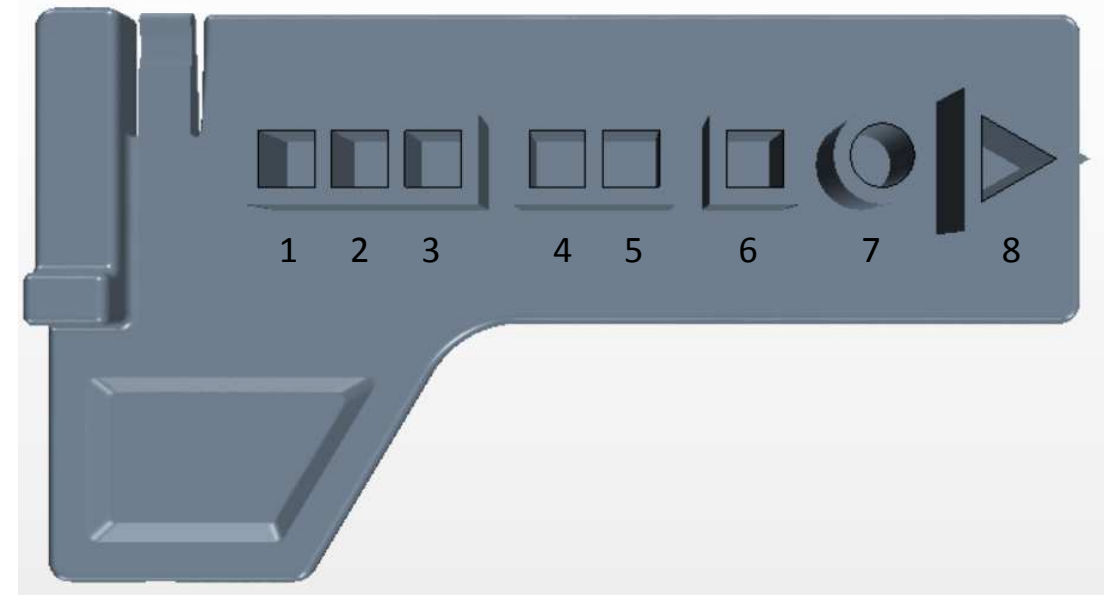

Figure 6.9-Single-piece 3-D printed CSELS CapEvap device

(a)



(b)
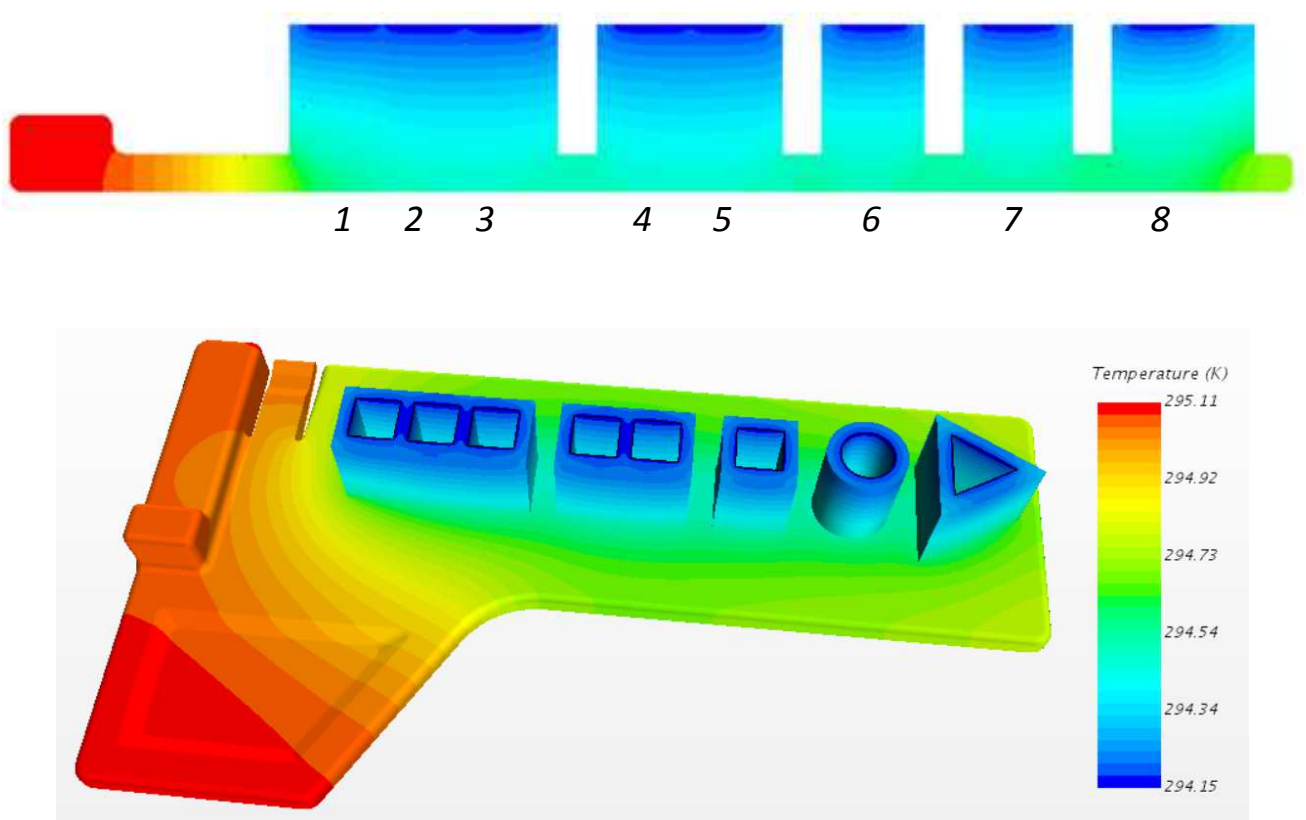

(c)

Figure 6.10 - (a): Heat flux to free surface, (b): Temperature profile mid-line of pores, (c): Temperature profile Pores and support 
A selection of images are provided in Figure 6.11 illustrating the variety of evaporation rates between pores for a single CSELS CapEvap test. All pores are initially $100 \%$ filled (i.e. initially flat interface). In this particular test complete evaporation is observed first from Pore 8 followed by Pores $3,6,1,4,5,7$, and 2 . This is somewhat confusing since though we might expect Pore 8 to be the fastest, we would expect Pores 1 and 3 to be identical, Pores 4 and 5 to be identical and Pore 7 to be the second slowest. Numerical heat transfer analysis sheds light on such outcomes. For example, the heat transfer numerical model is employed for the $100 \%$ filled condition which neglects changes in surface area and contact line length, between pores, to differing degrees, that occur during evaporation.

Observation of the time lapse images in Figure 6.11 shows that at least one of the left corners in Pore 3 (Figure 6.11 - (e)) remains wetted during almost the entire evaporation process. As with the triangle we now know that this mechanism is responsible for faster evaporation rates and likely explains the discrepancy observed for Pore 3. If such inadvertent wetting did not occur, Pore 3 would have required a much longer time to dry out. Furthermore, Pore 7 is observed to have the second longest evaporation time in Table 6.6, while also having the third highest measured heat flux. This discrepancy can be understood due to the $100 \%$ fill initial condition applied by the model which is not indicative of the entire evaporation process. As evaporation occurs the free surface area increases less for the circular pore than for the square and triangle pores and 
the contact line length for the circular pore remains constant. As a result we expect the circular pore to evaporate slower than the square and triangle as surface areas and contact line regions develop.

Table 6.6-CSELS Pore evaporation times and CFD heat transfer model results

\begin{tabular}{|c|c|c|}
\hline Cell & $\begin{array}{l}\text { Total time to evaporate } \\
\text { (Minutes) }\end{array}$ & CFD Heat Flux (W) \\
\hline 1 & 1964 & $8.32 \mathrm{E}-04$ \\
\hline 2 & $>2170$ & 7.00E-04 \\
\hline 3 & 1600 & $7.48 \mathrm{E}-04$ \\
\hline 4 & 2064 & 7.35E-04 \\
\hline 5 & 2170 & 7.36E-04 \\
\hline 6 & $>2170$ & 8.90E-04 \\
\hline 7 & 1660 & 8.46E-04 \\
\hline 8 & 1252 & 10.70E-04 \\
\hline
\end{tabular}




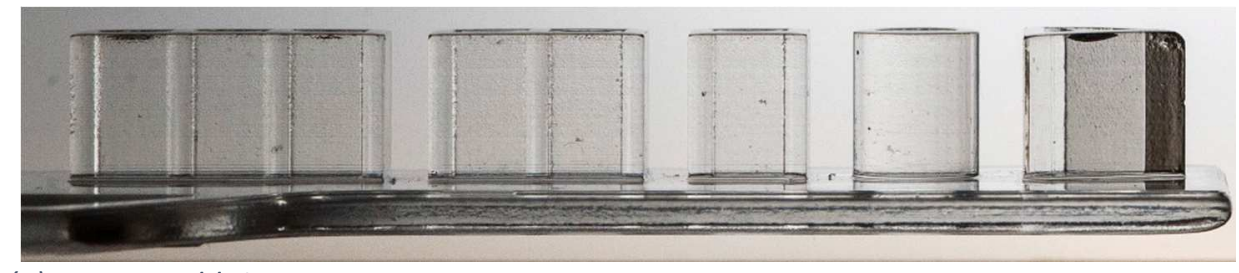

(a) 1 -> to add times...

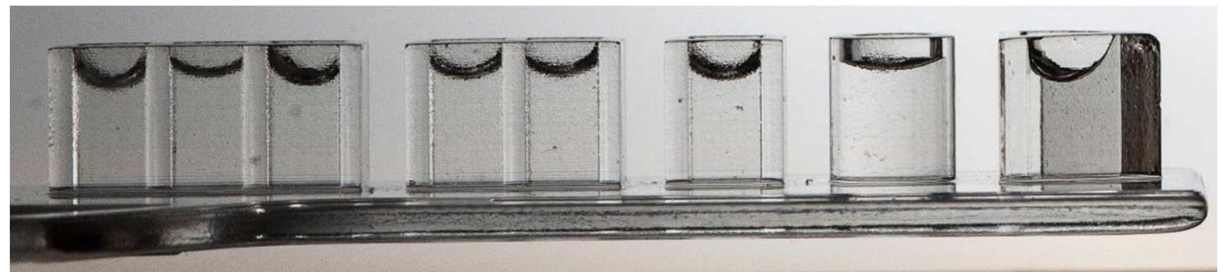

(b) 97

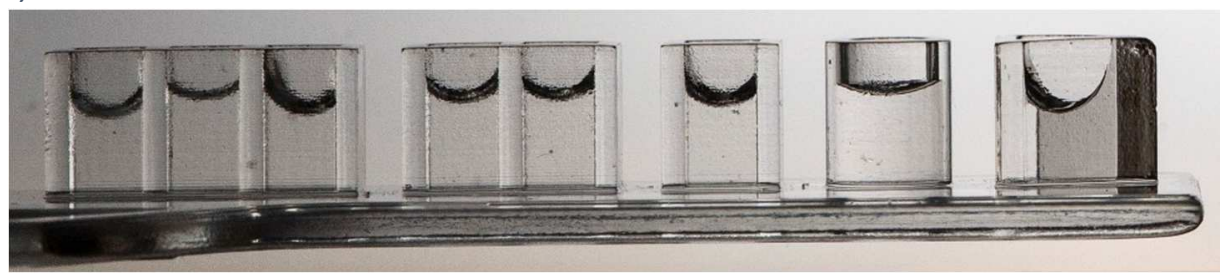

(c) 205

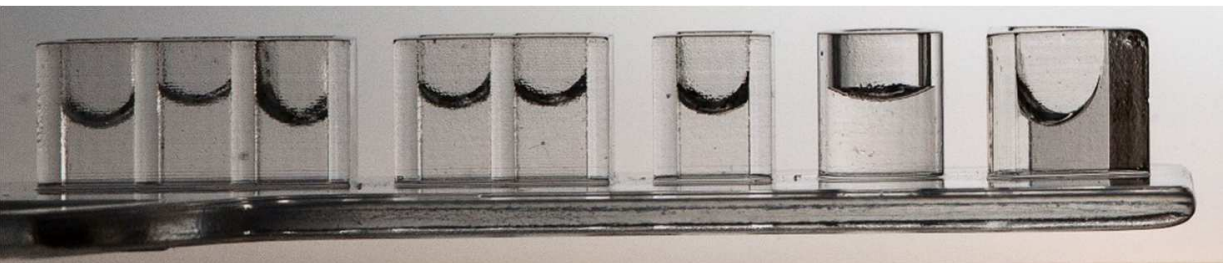

(d) 301

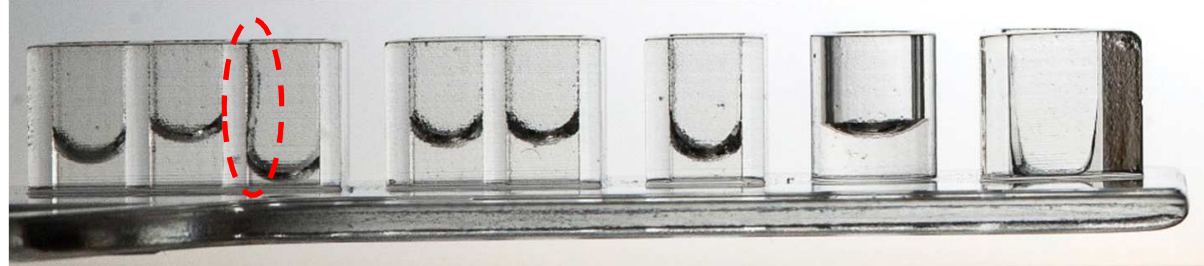

(e) 601

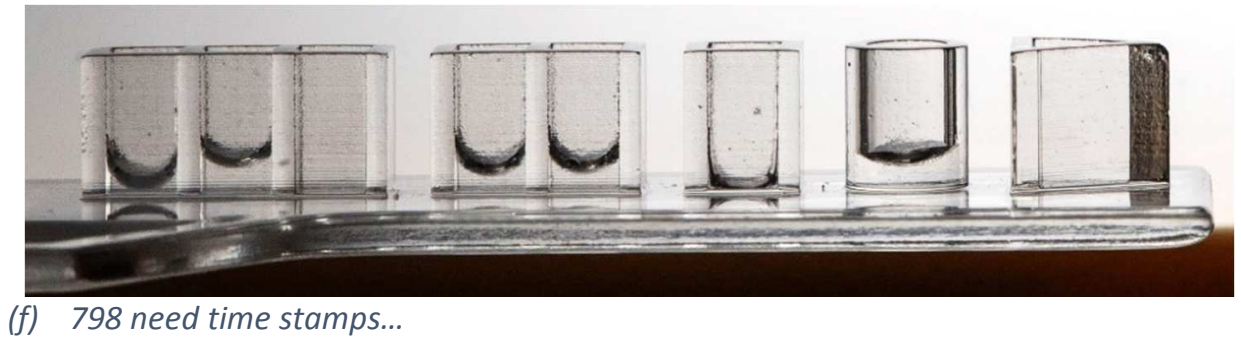

Figure 6.11 - Time lapse pores from Figure 1.1 (b) undergoing evaporation 


\subsection{Runtime}

All simulations were performed on the COEUS cluster at Portland State University. As can be seen in Table 6.1, run times vary considerably, ranging from 23.8 to 486 hours. The factors that most affect run times are the number of nodes in the mesh, and the time necessary for a process to achieve a quasi-steady state. Table 6.1 provides the shortest and longest run times encountered for the three types of models presented in this work: Stefan tube benchmark, CapEvap CSELS foam block evaporation, and CapEvap CSELS pore evaporation, as well as the number of cores used and the total CPU hours which represents the run time multiplied by the number of cores. The models requiring the longest times were the CapEvap CSELS pore evaporation models: i.e., 486 hours on an average of 300 cores and 150 hours on an average of 355 cores. Converting the run times above to a typical quad core computer, run times would range from approximately two to four years for the longest model. Because of the run times necessary for modelling the flows of interest in this research, High Powered Computing (HPC) is a necessity.

Table 6.7-Run time and number of cores used in simulations

\begin{tabular}{|c|c|c|c|}
\hline & Number of cores & $\begin{array}{l}\text { Total Solver Time } \\
\text { (Hours) }\end{array}$ & $\begin{array}{l}\text { CPU Solver Time } \\
\text { (Hours) }\end{array}$ \\
\hline Benchmark & 20 & $16.6-33.4$ & $330-743$ \\
\hline Pores (CSELS) & $300-400$ & $150-486$ & $53,217-146,272$ \\
\hline Foam (CSELS) & 100 & $23.8-71$ & $2141-7085$ \\
\hline
\end{tabular}




\section{Chapter 7}

\section{Conclusion}

The ability to quantitatively numerically model evaporation in microgravity is appealing as it affords the engineer a great degree of insight into the processes of interest as well as potentially reduce the time and cost of engineering system design. The numerical results help to describe mechanisms of interest that may otherwise be difficult to isolate from experimental measurements alone. The results from the ISS CSELS CapEvap experiments show that both pore geometry, gravity level, wetting properties, and air flow have significant impacts on the observed rates of evaporation. However, the CapEvap experiments do not illuminate the underlying causes. For example, the CapEvap triangular geometry completely evaporated within approximately 1,260 minutes while the square required approximately 1,660 minutes. The raw data shows that both geometries exhibit two approximately linear regions of evaporation (Figure 6.7). The initial evaporation rates for both geometries are similar when both menisci are essentially 
flat. However, as significant free surface curvature develops, contact line lengths increase, the menisci deepen, recede into the pores, and both geometries settle into different, approximately linear evaporation rates. In these linear regions, despite identical crosssectional areas, the triangle geometry evaporation rate is approximately 1.58 times greater than the square geometry for reasons that are now easily explained. The CapEvap experiments also captured significant differences in evaporation rates between 1-go and microgravity conditions. Experimental data for the CapEvap foam block shows that the evaporation rate in $1-g_{0}$ is approximately 1.53 times faster than the evaporation rate in microgravity. Such differences are attributed to airflow velocity to a known degree.

The viability of Star CCM+ to accurately model the CapEvap experiments is established by benchmarking the code against the Stefan Tube analytical solution (Figure 5.2). Four fill levels are chosen and computed to establish a quasi-steady evaporation rate. The evaporation rates are compared via Eq. 3.29 and agree with the analytical solution to within an average of $\pm 1 \%$.

Table 6.1 demonstrates that Star CCM+ is capable of capturing the evaporation rates from the square and triangular pore geometries to within $\pm 14 \%$ when comparing linear evaporation regions. Using the results generated in the CFD models allows the isolation of mechanisms responsible for differences in evaporation observed between the two geometries. For example, the numerical models show that the evaporation rates at the contact line regions are higher than at the bulk meniscus, even when the free surface 
is at a constant height (flat). While increasing contact line length contributes more to evaporation rate than does increasing the free surface area, the primary contributing factor, as shown in Figure $6.2(a)$, is the elevation of the free surface. As the free surface is maintained at higher elevations the evaporating liquid is exposed to lower humidity and greater air flow. This difference becomes more pronounced as the liquid recedes further into the pores. If the pores are deep enough, the square pore will stop evaporating altogether but the triangle pore will eventually reach a constant evaporation rate. This is due to the critically wetted interior corners of the triangular geometry that continuously pumps liquid to the opening of the pore where local humidity gradients are higher.

The Stefan Tube is idealized in that a uniform free surface height and a constant temperature are assumed. Results from the numerical models show that at a pore fill level of $75 \%$ the assumption of a uniform temperature is reasonable as the largest temperature drop for the triangular geometry is $0.89 \mathrm{~K}$ and $0.27 \mathrm{~K}$ for the square geometry. However, the free surface height varies more significantly. When the free surface in the square geometry has fully developed, the difference from the maximum height to the minimum height is $\sim 2 \mathrm{~mm}$ which in a pore with a total depth of $10 \mathrm{~mm}$ is not insignificant. Weighting the Stefan Tube both by actual area and actual contact line length increases the accuracy of the Stefan Tube equation for predicting evaporation rates observed in the CapEvap pores. However, weighting by the contact line length (Eq. 3.32) proves to be most accurate, which is in agreement with the results of Figure 6.8. Since evaporation 
rates are highest in the contact line region. Computed evaporation rates in the linear region are accurate to within $4.4 \%$ for the square pore and $6.1 \%$ for the triangular pore.

Table 6.2 shows how the evaporation rates vary significantly due to gravity and airflow rate. At an airflow of $1 \mathrm{~cm} / \mathrm{s}$ there is a difference of $60 \%$ in the evaporation rates between $1-g_{0}$ and microgravity captured in the models as well as $15 \%$ at $5 \mathrm{~cm} / \mathrm{s}$. These differences can be attributed to a lack of buoyancy in the vapor phase under microgravity conditions. However, the differences in evaporation rates between $1-g_{0}$ and microgravity readily diminish with increased airflow velocity. With a sufficiently high velocity the differences in evaporation rates due to gravity are eliminated entirely. Because the surface temperatures are nearly uniform and approximately the same for the four models presented in Table 6.3, this evaporation process can be approximated as a heat transfer problem to establish the contributions of forced versus natural convection characterized by the Richardson number, $R i$, of which the results are plotted in Figure 6.6. We observe that at a velocity of $1 \mathrm{~cm} / \mathrm{s}$, neither natural or forced convection can be neglected. However, with an air speed of only $5 \mathrm{~cm} / \mathrm{s}$, forced convection is dominant and the impact of natural convection is negligible in agreement with the results from the numerical models.

The numerical results presented herein shed light on the mechanisms affecting evaporation rates aboard spacecraft and provide methods to effectively model them. By understanding the factors that limit evaporation rates in microgravity, engineers can 
better optimize processes to minimize them. The applications-potential of the current twice-benchmarked numerical tool is high in that further computations can be pursued varying pore geometry, pore interconnectivity, fluid properties, and cabin ambient conditions such as relative humidity, temperature, pressure, and airflow velocity and direction. An application problem is solved numerically for the global heat transfer of a single CSELS CapEvap experiment where the various evaporation rates of numerous pores are explained with good confidence. 
References:

[1] Steven Siceloff, 11.17.2008, Recycling Water is not Just for Earth Anymore, https://www.nasa.gov/mission pages/station/behindscenes/waterrecycler.html, 01.20 .2020

[2] Hiroshi Nomura, Takahiro Murakoshi, Yusuke Suganuma, Yasushige Ujiie, Nozomu Hashimoto, AND Hiroyuki Nishida, Microgravity Experiments of fuel droplet evaporation in sub and supercritical environments, Proceedings of the Combustion Institute, 36 (2017), pp. 2246-2432

[3] Pensinger, S., M.R. Callahan, M.M. Weislogel, K. Viestenz, M. Campbell, Development of a Foam-Based Capillary-Driven Brine Residual in Containment (BRIC) Processor,47th Int. Conf. Environ. Sys., ICES-2017-350, 16-20 July 2017, Charleston, South Carolina.

[4] Hirt, C.W ; Nichols, B.D, Volume of fluid (VOF) method for the dynamics of free boundaries, Journal of Computational Physics, 1981, 39 (1981), pp.201-225

[5] Chen, Y., B. Schaffer, M. Weislogel, G. Zimmerli, Introducing SE-FIT: Surface Evolver Fluid Interface Tool for Studying Capillary Surfaces, AIAA 2011-1319, 49th AIAA Aerospace Sci. Mtg, Orlando, Jan. 2011: https://doi.org/10.2514/6.2011-1319

[6] Kenneth A. Brakke, The Surface Evolver, Experimental Mathematics, Vol. 1 (1992)

[7] Antoine, C., Vapor Pressure: a new relationship between pressure and temperature, Comptes Rendus des Séances de l'Académie des Sciences (1888), 107: 681-684, 778-780, 836-837

[8] J. Stefan, Über das Gleichgewicht und die Bewegung, insbesondere die Diffusion von Gasgemengen Sitzungsber. kais. Akad. Wiss. Wien. Math. Naturwiss. Kl., Abt. II a, 63 (1871), pp. 63-124

[9] Courant, R., Friedrichs, K., AND Lewy, H., Über die partiellen Differenzengleichungen der mathematischen Physik, Mathematische Annalen (1928), 100, pp. 32-74

[10] J.U. Brackbill, D.B. Kothe, AND C. Zemach, A Continuum Method for Modeling Surface Tension, Journal of Computational Physics (1992), 100, pp. 335-354

[11] Concus, P., Finn, R. On the Behavior of a Capillary Surface in a Wedge. Appl. Math. Sci. 1969, 63, 292-299. 